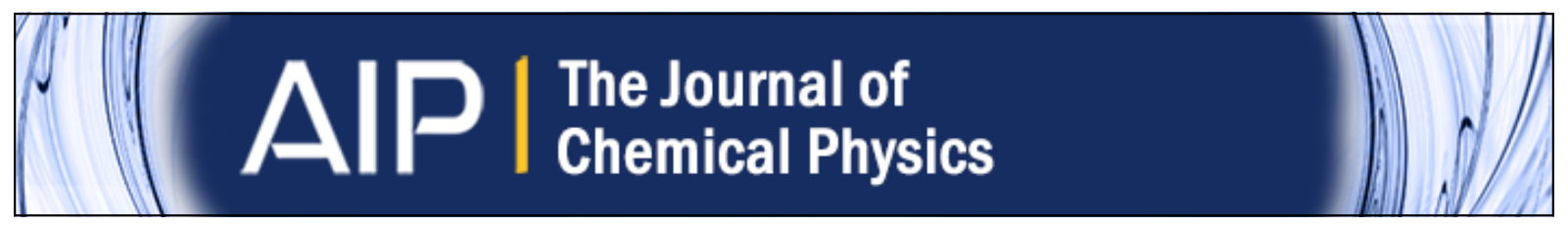

\title{
Electronic excitations in long polyenes revisited
}

Maximilian Schmidt and Paul Tavan

Citation: The Journal of Chemical Physics 136, 124309 (2012); doi: 10.1063/1.3696880

View online: http://dx.doi.org/10.1063/1.3696880

View Table of Contents: http://scitation.aip.org/content/aip/journal/jcp/136/12?ver=pdfcov

Published by the AIP Publishing

Advertisement:

\section{AlP Re-register for Table of Content Alerts}




\title{
Electronic excitations in long polyenes revisited
}

\author{
Maximilian Schmidt and Paul Tavan a) \\ Lehrstuhl für BioMolekulare Optik, Ludwig-Maximilians Universität München, \\ Oettingenstr. 67, 80538 München, Germany
}

(Received 2 November 2011; accepted 6 March 2012; published online 27 March 2012)

\begin{abstract}
We apply the valence shell model OM2 [W. Weber and W. Thiel, Theor. Chem. Acc. 103, 495, (2000)] combined with multireference configuration interaction (MRCI) to compute the vertical excitation energies and transition dipole moments of the low-energy singlet excitations in the polyenes with $4 \leq N \leq 22 \pi$-electrons. We find that the OM2/MRCI descriptions closely resemble those of Pariser-Parr-Pople (PPP) $\pi$-electron models [P. Tavan and K. Schulten, Phys. Rev. B 36, 4337, (1987)], if equivalent MRCI procedures and regularly alternating model geometries are used. OM2/MRCI optimized geometries are shown to entail improved descriptions particularly for smaller polyenes $(N \leq 12)$, for which sizeable deviations from the regular model geometries are found. With configuration interaction active spaces covering also the $\sigma$-in addition to the $\pi$-electrons, OM2/MRCI excitation energies turn out to become smaller by at most $0.35 \mathrm{eV}$ for the ionic and $0.15 \mathrm{eV}$ for the covalent excitations. The particle-hole $(p h)$ symmetry, which in Pariser-Parr-Pople models arises from the zero-differential overlap approximation, is demonstrated to be only weakly broken in OM2 such that the oscillator strengths of the covalent $1 \mathrm{~B}_{\mathrm{u}}^{-}$states, which artificially vanish in $p h$-symmetric models, are predicted to be very small. According to OM2/MRCI and experimental data the $1 \mathrm{~B}_{\mathrm{u}}^{-}$state is the third excited singlet state for $N<12$ and becomes the second for $N \geq 14$. By comparisons with results of other theoretical approaches and experimental evidence we argue that deficiencies of the particular MRCI method employed by us, which show up in a poor size consistency of the covalent excitations for $N>12$, are caused by its restriction to at most doubly excited references. () 2012 American Institute of Physics. [http://dx.doi.org/10.1063/1.3696880]
\end{abstract}

\section{INTRODUCTION}

Based on multireference configuration interaction (MRCI) calculations for the Pariser-Parr-Pople ${ }^{1-3}$ (PPP) and Hubbard ${ }^{4}$ models of polyenes (see Fig. 1 for the chemical structure of these linearly conjugated molecules) as well as using analytical results on the infinite Hubbard chain, 5,6 Tavan and Schulten published in 1987 a seminal paper on the "Electronic excitations in finite and infinite polyenes."7 In view of the rather simple $\pi$-electron model Hamiltonians employed in this study, its apparent and lasting success is surprising. Considering the substantial progress in computer technology, in the efficiency of configuration interaction (CI) programs (see, e.g., Ref. 8) and in the development of improved valence shell Hamiltonians particularly achieved by the so-called OM2 model, $,{ }^{9}, 10$ a reinvestigation of this issue seems worthwhile and is the purpose of this contribution.

\section{A. Theory of $\pi$-electron excitations in long polyenes}

Although the quoted paper ${ }^{7}$ in part solely exploited a previous PPP/MRCI prediction ${ }^{11}$ on the energetic ordering of the electronically excited singlet states $S_{i}, i=1,2, \ldots$ in regularly alternating polyenes $N=4,6, \ldots, 16 \pi$-electrons, it added important insights, e.g., by relating the excitation energies $\Delta E_{i}(N)$ to two different dispersion relations $E^{\beta}(k)$, of quasiparticles $\beta=1,2$ in the infinite system, i.e., polyacetylene.

\footnotetext{
a)Electronic mail: tavan@physik.uni-muenchen.de.
}

Two classes of quasi-particle excitations $\beta$ were identified, the triplet-triplet $(t t)$ magnons and the particle-hole $(p h)$ excitons. As explained in more detail in Sec. I A of the supplementary material $^{12}(\mathrm{SM})$, each excited state $S_{i}$ in a polyene of size $N$ is characterized by the quasi-particle label $\beta$ and by a momentum quantum number $q=1,2, \ldots$, which defines the discrete quasi-particle momentum $k_{q}(N)=q \pi /(N+1)$. Then the excitation energies derive through $\Delta E_{q}^{\beta}(N)=E^{\beta}\left(k_{q}(N)\right)$. This connection is exact in one-electron theory. In manyelectron theory it becomes exact in the limit of large systems $(N \rightarrow \infty){ }^{13}$

The three lowest covalent ${ }^{11,14,15}$ excited singlet states of the polyenes were shown to belong to the class of $t$ magnons. ${ }^{7}, 13$ Here, the name "covalent" derives from the dominant covalent Rumer diagrams appearing in valence bond descriptions (see, e.g., Refs. 16-18). The covalent excitations are the $2 \mathrm{~A}_{\mathrm{g}}^{-}, 1 \mathrm{~B}_{\mathrm{u}}^{-}$, and $3 \mathrm{~A}_{\mathrm{g}}^{-}$states as classified by the irreducible representations of the $C_{2 \mathrm{~h}}$ point groups of the all-trans polyenes and by the "Pariser alternancy symmetry" (PAS), which provides the additional labels "+" and "-.." ${ }^{14,19}$ The PAS is an artificial symmetry appearing in $\pi$ electron models such as Hückel, ${ }^{20}$ PPP, or Hubbard, which assume a zero-differential overlap (ZDO) between the atomic $p_{z}$ orbitals. In correlated ZDO models the covalent singlet states of the polyenes are all characterized by the PAS label "-.."

On the other hand, the two lowest ionic ${ }^{11,14,15} \pi-\pi^{*}$ excitations $1 \mathrm{~B}_{\mathrm{u}}^{+}$and $1 \mathrm{~A}_{\mathrm{g}}^{+}$of the finite chains belong to the dispersion $E^{p h}(k)$ of the $p h$-excitons. ${ }^{7,13}$ All ionic states are characterized by the PAS label "+." 


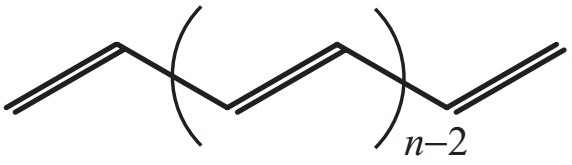

FIG. 1. Chemical structure of all-trans polyenes $\mathrm{H}(\mathrm{CH}=\mathrm{CH})_{n} \mathrm{H}$, with $N=2 n \pi$-electrons

Adding the PAS labels "+" or "-" to the group theoretical state symbols is common in polyene spectroscopy ${ }^{16,21-23}$ although the PAS is an exclusive feature of ZDO models and is absent in real polyenes. According to the PAS, transitions between states of identical PAS symmetry (such as the $1 \mathrm{~A}_{\mathrm{g}}^{-}$ground state and the $1 \mathrm{~B}_{\mathrm{u}}^{-}$excited state) are strictly dipole-forbidden and, correspondingly, many authors erroneously were misled to think that this peculiar property of ZDO models is valid also in actual polyenes (see, e.g., the discussions on p. 2397 in Ref. 24). However, since 1998 several authors $^{25-27}$ have demonstrated that the $1 \mathrm{~B}_{\mathrm{u}}^{-}$states actually carry small transition dipole moments.

Because the description of the diatomic overlap has been greatly improved in the OM2 valence shell Hamiltonian, which has been developed and carefully evaluated by Thiel and co-workers for a series of different dye molecules and various levels of $\mathrm{CI},{ }^{9,10}$ we expect that our following OM2/MRCI treatment can properly account for the excitation energies and oscillator strengths of the covalent and ionic excitations in the polyenes.

Figure 2 illustrates the early computational results ${ }^{7,11} \mathrm{ob}-$ tained by PPP-MRCI calculations on the vertical excitation energies $\Delta E_{i}(N)$ of the low-lying singlet states $S_{i}$ in regularly alternating polyenes with $N=4,6, \ldots, 16 \pi$-electrons. In particular, these results predicted that the $1 \mathrm{~B}_{\mathrm{u}}^{+}$state, which causes the strong optical absorption band of the all-trans polyenes and is the second excited singlet state $S_{2}$ in shorter polyenes, ${ }^{16}$ becomes the third excited singlet state $S_{3}$ in long polyenes ( $N$ $\gtrsim 10$ ), because the excitation energy of the lowest covalent state of $\mathrm{B}_{\mathrm{u}}$ symmetry $\left(1 \mathrm{~B}_{\mathrm{u}}^{-}\right)$decreases more steeply with increasing polyene length than that of its ionic relative $\left(1 \mathrm{~B}_{\mathrm{u}}^{+}\right)$.

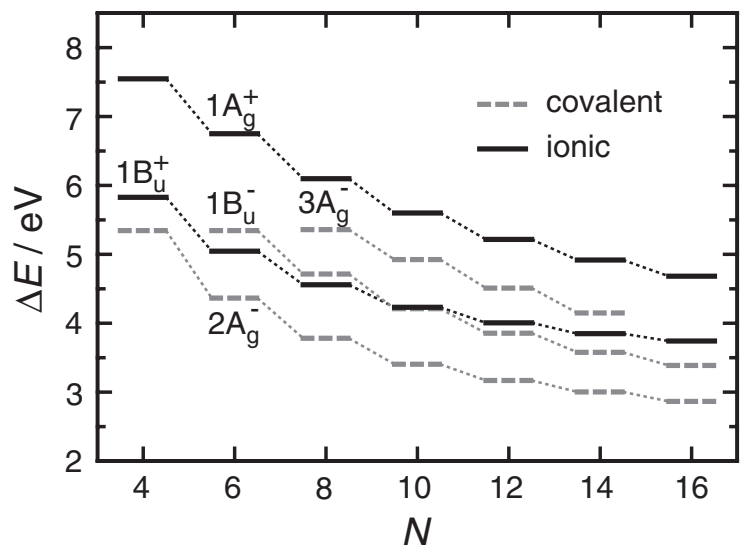

FIG. 2. Excitation energies $\Delta E(N)$ of the low-lying ionic $\left(1 \mathrm{~B}_{\mathrm{u}}^{+}, 1 \mathrm{~A}_{\mathrm{g}}^{+}\right)$and covalent $\left(2 \mathrm{~A}_{\mathrm{g}}^{-}, 1 \mathrm{~B}_{\mathrm{u}}^{-}, 3 \mathrm{~A}_{\mathrm{g}}^{-}\right)$singlet states in polyenes with $4 \leq N \leq 16$ $\pi$-electrons from early PPP-MRCI calculations. ${ }^{7,11}$
Similarly, the $3 \mathrm{~A}_{\mathrm{g}}^{-}$state and higher $t t$-excitations should also fall below the $1 \mathrm{~B}_{\mathrm{u}}^{+}$state in sufficiently long polyenes. ${ }^{7}$

\section{B. Development of polyene spectroscopy}

Concerning polyene spectroscopy, the prediction that an increasing number of covalent $t$-excitations should appear in the energy gap $\Delta E_{\mathrm{BA}} \equiv E\left(1 \mathrm{~B}_{\mathrm{u}}^{+}\right)-E\left(2 \mathrm{~A}_{\mathrm{g}}^{-}\right)>0$ between the optically bright $1 \mathrm{~B}_{\mathrm{u}}^{+}$state and the lowest covalent excitation $2 \mathrm{~A}_{\mathrm{g}}^{-}$in long polyenes was the key result of Ref. 7, which distinguished this theory from earlier ones and from the status of experimental knowledge available at that time. Since the early 1970s the excited state sequences $S_{1} \equiv 2 \mathrm{~A}_{\mathrm{g}}^{-}$and $S_{2}$ $\equiv 1 \mathrm{~B}_{\mathrm{u}}^{+}$had been firmly established theoretically and experimentally for short polyenes $(N \lesssim 10)$, the finding of which had led to the assumption that this sequence of singlet states holds for all polyenes. ${ }^{16,28,29}$ Note, however, that this sequence is still a matter of debate ${ }^{30-33}$ for the shortest polyenes with $N \leq 8$ (cf. Sec. I B in the SM).

It took more than a decade until the quoted prediction was verified experimentally ${ }^{24,34}$ and it has been frequently emphasized that the intermediate covalent states of the carotenoids (e.g., $1 \mathrm{~B}_{\mathrm{u}}^{-}, 3 \mathrm{~A}_{\mathrm{g}}^{-}$) can support the efficient energy transfer to the chlorophylls in the light harvesting process of photosynthesis. ${ }^{21,23,35}$

It may be, however, that the presence of the $1 \mathrm{~B}_{\mathrm{u}}^{-} \sin -$ glet state below the $1 \mathrm{~B}_{\mathrm{u}}^{+}$state has been actually observed in pure polyenes much earlier. In 1985 Snyder et al. ${ }^{36}$ interestingly observed a drastic qualitative change of the polyene fluorescence spectrum upon increasing the size of an all-transpolyene from $N=12 \pi$-electrons to $N=14$. They attributed a fluorescence newly appearing at $N=14$ energetically a little below the onset of the $1 \mathrm{~B}_{\mathrm{u}}^{+}$absorption to this state despite the $370 \mathrm{~cm}^{-1}$ Stokes shift and the lack of symmetry between the absorption and emission spectrum, both of which are easily rationalized in terms of another electronic state $\left(1 \mathrm{~B}_{\mathrm{u}}^{-}\right)$slightly below the $1 \mathrm{~B}_{\mathrm{u}}^{+}$state. According to Wang et al. ${ }^{37}$ the $1 \mathrm{~B}_{\mathrm{u}}^{-}$state appears below the $1 \mathrm{~B}_{\mathrm{u}}^{+}$state in the carotenoids for $N>14$, i.e., at a size very close to that of the pure polyene studied in Ref. 36. Thus, the 0-0 transitions of the two $B_{u}$ states appear to switch their respective energetic ordering somewhere above $N=10$ in agreement with the early PPP/MRCI results displayed by Fig. 2, which however refer to model geometries and vertical transitions.

Note here that the SM (Ref. 12) provides in Table S4 experimental (gas phase) polyene excitation energies constructed and compiled by us from many sources available in the literature. ${ }^{36-42}$ Additionally, these data are graphically represented in the accompanying Figs. S15 and S16.

\section{Quantum chemical descriptions of polyene spectra}

The apparent success of the quoted PPP/MRCI descriptions ${ }^{7,11}$ has subsequently inspired several theory groups to search for additional covalent excitations energetically below the bright $1 \mathrm{~B}_{\mathrm{u}}^{+}$absorption in long polyenes $(N \geq 12)$ using more advanced theoretical methods. ${ }^{26,43-45}$ 
For instance, in 2004 Kurashige et al. ${ }^{43}$ studied the alltrans-polyenes with $6 \leq N \leq 28 \pi$-electrons in a correlated $a b$ initio setting, which restricted the active space for full CI expansions to the five highest occupied and five lowest unoccupied molecular orbitals (MOs), and estimated $\sigma-\pi$ interactions by second order perturbation theory. According to these calculations the vertical transition to the $1 \mathrm{~B}_{\mathrm{u}}^{-}$state appears below the $1 \mathrm{~B}_{\mathrm{u}}^{+}$transition at $N \geq 14$ and the transition to the $3 \mathrm{~A}_{\mathrm{g}}^{-}$state at $N \geq 22$. In the carotenoids the latter state switch is observed for the $0-0$ transitions at $N \geq 20,{ }^{37}$ while the early PPP/MRCI treatment yielded an extrapolated switch at $N \geq 20$.

Using various $a b$ initio basis sets and a correlation treatment applying the "extended second order diagrammatic construction" $[\mathrm{ADC}(2)-\mathrm{x}]$, Starcke et $a .^{44}$ considered the polyenes with $4 \leq N \leq 12$. Their calculations predicted not only the $2 \mathrm{~A}_{\mathrm{g}}^{-}$state but also the $1 \mathrm{~B}_{\mathrm{u}}^{-}$state for all polyenes with $N \geq 6$ below the transition to the ionic $1 \mathrm{~B}_{\mathrm{u}}^{+}$state. Similarly, the "density matrix renormalization group" approach (DMRG), which was combined with complete active space self-consistent field (CASSCF) calculations, of Ghosh et al. ${ }^{26}$ which were carried out for polyenes with $N=8,12,16,20,24$ $\pi$-electrons, placed already at $N=8$ the covalent excitations $2 \mathrm{~A}_{\mathrm{g}}^{-}, 1 \mathrm{~B}_{\mathrm{u}}^{-}$, and $3 \mathrm{~A}_{\mathrm{g}}^{-}$below the optical transition.

Finally, Marian and Gilka ${ }^{45}$ applied the "density functional theory multireference configuration interaction" method (DFT/MRCI) to polyenes with $6 \geq N \geq 26$ using extended basis sets, geometries optimized for single determinant Kohn-Sham ground states, and described dynamic correlations by MRCI expansions "kept short by extensive configuration selection at a few thousand configuration state functions." ${ }^{46}$ According to these results the $1 \mathrm{~B}_{\mathrm{u}}^{-}$state becomes the $S_{2}$ state for $N \gtrsim 22$ at the chosen Franck-Condon geometry, while the $3 \mathrm{~A}_{\mathrm{g}}^{-}$singlet excitation stays above the $1 \mathrm{~B}_{\mathrm{u}}^{+}$state in all polyenes considered.

In summary, the quoted calculations ${ }^{26,43-45}$ and, here, particularly Refs. 43 and 45, essentially confirmed the early PPP/MRCI descriptions of the low-lying electronically excited singlet states concerning the relative spacings of the covalent and ionic states in the polyenes. In contrast, conventional and very expensive correlated $a b$ initio treatments based, e.g., on third order coupled cluster theory, ${ }^{47}$ which are applicable only to relatively small polyenes, tend to invert the experimental state order by assigning negative gaps $\Delta E_{\mathrm{BA}}$ of $-0.19 \mathrm{eV},-0.14 \mathrm{eV}$, and $-0.03 \mathrm{eV}$ for $N=4,6$, and 8 , respectively [for discussions of this issue see Ref. $48(N=4)$ and Sec. I B of the SM $(N=8)$ ].

\section{Open questions}

In view of the well-established theory of polyene spectra outlined above it may seem that a renewed study of the polyene spectra using a combination ${ }^{8}$ of extended CI with a valence shell model such as $\mathrm{OM} 2^{9,10}$ cannot add important insights. In fact, concerning the excited state orderings in long polyenes we cannot expect much more than a confirmation of previous results. However, concerning other issues OM2/MRCI can open new perspectives.
First, it enables an answer to the important theoretical question why the PPP/MRCI descriptions were so successful despite the use of model geometries instead of optimized geometries and despite the inherent neglect of $\sigma-\pi$ interactions. Here, it will allow us to check how strongly the excitation energies change, if relaxed ground state geometries are used and if $\sigma-\pi$ (and $\sigma-\sigma$ ) correlations are included. Second, by comparison with carefully compiled experimental data (cf. Sec. I B in the SM) it will indicate which type of MRCI approach is necessary for high-quality OM2 calculations of polyene excited states. Third, it will yield transition dipoles for the $1 \mathrm{~A}_{\mathrm{g}}^{-} \rightarrow 1 \mathrm{~B}_{\mathrm{u}}^{-}$excitations in long polyenes and, thereby, indicate to what extent the PAS (Refs. 14, 19) is actually broken in OM2/MRCI. These issues represent the program of our current contribution. In a subsequent contribution we intend to address the geometry relaxations occurring in the various excited singlet states thus getting access to $0-0$ transitions.

\section{METHODS}

We carried out large scale CI calculations on the polyene spectra using the OM2 valence shell model Hamiltonian ${ }^{9}$ and, for comparison with previous CI descriptions of the correlated $\pi-\pi *$ excitations, ${ }^{7,11}$ also with the PPP $\pi$-electron model. ${ }^{1-3}$ We applied the PPP Hamiltonian in the form and with the parameters employed previously., ${ }^{71}$

\section{A. Cl methods}

The CI expansions were made up from spin adapted configurations (SACs). The associated CI matrices were calculated using the matrix element formulas of Tavan and Schulten ${ }^{49}$ combined with the algorithm for efficient evaluation and compact storage of CI matrices of Strodel and Tavan. ${ }^{8}$ The corresponding CI program offers, in addition to PPP, an interface to the MNDO97 (Ref. 50) program package, and therefore enables also OM2/CI calculations. ${ }^{8,48}$

Disregarding the possibilities of individual configuration selection, in the following OM2/CI study we either included all SACs, which are singly and doubly excited with respect to the closed shell Hartree-Fock (HF) ground state (DCI), or all SACs, which are additionally triply and quadruply excited (QCI). Furthermore we carried out MRCI calculations, in which the reference SACs were selected for each excited state from the DCI wave function according to certain selection criteria.

\section{B. Active HF-MO spaces}

The "active" space of HF MOs included into CI consisted in the case of PPP of all $\pi$-MOs. In the case of OM2 we selected certain MO subsets to keep the CI expansions handily small. Here we used either all $N \pi$-orbitals and called a corresponding calculation "OM $2 / \mathrm{CI}(\pi)$," or we added certain sets of $\sigma$-orbitals. We chose either all $4 N+2 \sigma$-orbitals ["OM2/CI $(\sigma, \pi)$ "] or only the subset consisting of the $N+1$ energetically highest occupied and the $N+1$ energetically lowest unoccupied HF-MOs ["OM2/CI $\left(\sigma_{r}, \pi\right)$ "]. The latter 
distinction is suggested by a peculiar band gap separating in the OM2 model of the polyenes the $N$ energetically lowest occupied and $N$ energetically highest unoccupied $\sigma$-orbitals from the remaining $\left(\sigma_{r}, \pi\right) \mathrm{MO}$ set just introduced. This gap is apparent in Fig. S17 provided in the SM.

\section{C. $\mathrm{MRCl}$ reference sets and SAC dimensions}

MRCI methods aim at a size-consistent description of excitation energies through a cancellation of the sizeextensiveness errors inherent to truncated $\mathrm{CI}$ expansions. The specification of a particular MRCI method requires the definition of a procedure for selecting the set $R(s, N)$ of reference SACs characterizing a given electronic state $s$ in a polyene with $N \pi$-electrons. The MRCI basis then consists of the set $R(s, N)$ and of all those SACs, which are singly and doubly excited with respect to the elements of $R(s, N)$. We chose two different procedures for the selection of the reference sets $R(s, N)$.

In a global approach we included for the ground state solely the closed shell HF determinant into $R\left(1 \mathrm{~A}_{\mathrm{g}}^{-}, N\right)$, while for the excited states the reference sets covered the complete DCI bases of the associated symmetries. Thus, the ground state is calculated by DCI and the excited states are calculated by QCI. We call this approach $\mathrm{MRCI}_{\mathrm{c}}$, because the reference sets are (on the DCI level) "complete" for all states.

In a state specific and more approximate approach we selected the excited state reference sets $R(s, N)$ from DCI calculations by including only those SACs into $R(s, N)$ which have the largest CI coefficients and whose cumulative contribution to the DCI wave function exceeds a certain threshold $\theta \cdot{ }^{11,48}$ We arbitrarily chose the value $\theta=92 \%$. We call the approach associated with this state specific reference selection "MRCI $\mathrm{s}_{\mathrm{s}}$." Like in $\mathrm{MRCI}_{\mathrm{c}}$ also here the set $R\left(1 \mathrm{~A}_{\mathrm{g}}^{-}, N\right)$ was restricted to the HF determinant. Therefore, differences of $\mathrm{MRCI}_{\mathrm{s}}$ and $\mathrm{MRCI}_{\mathrm{c}}$ excitation energies solely derive from excited state energies.

For the PPP Hamiltonian of the polyenes with $4 \leq N$ $\leq 22 \pi$-electrons our DCI based MRCI s $_{\mathrm{s}}$ reference selection procedure yields reference sets $R(s, N)$, whose sizes $|R(s, N)|$ are listed in Table I. For the excited states the threshold $\theta=92 \%$ leads to numbers $|R(s, N)|$, which increase with $N$.

TABLE I. Numbers of references $|R(s, N)|$ used in PPP/MRCI for different polyenes $N$. For the excited states the reference SACs in the sets $R(s, N)$ cover at least $92 \%$ of the DCI wave functions.

\begin{tabular}{lcrrr}
\hline \hline$N$ & $1 \mathrm{~A}_{\mathrm{g}}^{-}$ & $2 \mathrm{~A}_{\mathrm{g}}^{-}$ & $1 \mathrm{~B}_{\mathrm{u}}^{-}$ & $1 \mathrm{~B}_{\mathrm{u}}^{+}$ \\
\hline 4 & 1 & 6 & - & 1 \\
6 & 1 & 11 & 9 & 1 \\
8 & 1 & 17 & 18 & 1 \\
10 & 1 & 23 & 31 & 2 \\
12 & 1 & 32 & 47 & 2 \\
14 & 1 & 48 & 66 & 2 \\
16 & 1 & 67 & 88 & 3 \\
18 & 1 & 86 & 118 & 4 \\
20 & 1 & 110 & 151 & 5 \\
22 & 1 & 141 & 194 & 6 \\
\hline \hline
\end{tabular}

TABLE II. PPP/CI SAC dimensions for the $1 \mathrm{~A}_{\mathrm{g}}^{-}$ground state, for the $\mathrm{A}_{\mathrm{g}}$ and $\mathrm{B}_{\mathrm{u}}$ excitations treated by $\mathrm{MRCI}_{\mathrm{c}}$, and for three low-lying excited states described by $\mathrm{MRCI}_{\mathrm{S}}$ at different polyene lengths $N$.

\begin{tabular}{|c|c|c|c|c|c|c|}
\hline \multicolumn{2}{|c|}{ MRCI } & \multicolumn{2}{|c|}{$\mathrm{MRCI}_{\mathrm{c}}$} & \multicolumn{3}{|c|}{$\mathrm{MRCI}_{\mathrm{s}}$} \\
\hline$N$ & $1 A_{g}^{-}$ & $A_{g}$ & $\mathrm{~B}_{\mathrm{u}}$ & $2 \mathrm{~A}_{\mathrm{g}}^{-}$ & $1 \mathrm{~B}_{\mathrm{u}}^{-}$ & $1 \mathrm{~B}_{\mathrm{u}}^{+}$ \\
\hline 4 & 9 & 12 & 9 & 12 & - & 9 \\
\hline 6 & 30 & 87 & 79 & 86 & 78 & 41 \\
\hline 8 & 81 & 611 & 585 & 557 & 525 & 130 \\
\hline 10 & 182 & 3328 & 3274 & 2471 & 2700 & 551 \\
\hline 12 & 361 & 14198 & 14081 & 8628 & 11271 & 1225 \\
\hline 14 & 650 & 49495 & 49291 & 26637 & 34572 & 2379 \\
\hline 16 & 1089 & 47273 & 146913 & 68547 & 89264 & 5933 \\
\hline 18 & 1722 & 386498 & 385938 & 164267 & 212574 & 16355 \\
\hline 20 & 2601 & 917076 & 916201 & 327127 & 438757 & 36138 \\
\hline 22 & 3782 & 1933501 & 2002647 & 680759 & 830266 & 61908 \\
\hline
\end{tabular}

This increase is much smaller for the ionic $1 \mathrm{~B}_{\mathrm{u}}^{+}$state than for the two covalent excitations. Among the latter it is more pronounced for the $1 \mathrm{~B}_{\mathrm{u}}^{-}$than for the $2 \mathrm{~A}_{\mathrm{g}}^{-}$state indicating that correlation effects are even more important for the higher covalent excitations than for the already highly correlated $2 \mathrm{~A}_{\mathrm{g}}^{-}$ state. Note that the $1 \mathrm{~B}_{\mathrm{u}}^{-}$state does not yet exist in butadiene $(N$ $=4$ ), because it is a simultaneous excitation of three $\pi$-bonds into three local triplets coupled to an overall singlet state ${ }^{7}$ and because butadiene has only two such bonds.

In $\mathrm{OM} 2 / \mathrm{MRCI}_{\mathrm{s}}(\pi)$ calculations one gets slightly smaller numbers of references (cf. Table S15 in the SM). The $\mathrm{OM} 2 / \mathrm{MRCI}_{\mathrm{s}}(\pi)$ excitation energies turned out to be very similar to those from the more expensive $\mathrm{MRCI}_{\mathrm{c}}$ approach, which is why we do not show them here.

Table II compares for the PPP/CI calculations the dimensions of the SAC expansions, which result for the MRCI description of the $1 \mathrm{~A}_{\mathrm{g}}^{-}$ground state, for the $\mathrm{MRCI}_{\mathrm{c}}$ expansions of the excited states with $A_{g}$ and $B_{u}$ symmetries, respectively, and for the $\mathrm{MRCI}_{\mathrm{S}}$ treatments of the three low-lying covalent and ionic excitations. Note here that we did not utilize the PAS to further reduce the dimensions of the CI matrices like in the earlier work. ${ }^{7,11}$ Therefore, the dimensions listed in the table also apply to $\mathrm{OM} 2 / \mathrm{CI}(\pi)$ descriptions, as long as the $1 \mathrm{~A}_{\mathrm{g}}^{-}$state and $\mathrm{OM} 2 / \mathrm{MRCI}_{\mathrm{c}}(\pi)$ are considered. For $\mathrm{OM} 2 / \mathrm{MRCI}_{\mathrm{s}}(\pi)$ the SAC dimensions (not shown) are similar to those given in the table for PPP/CI.

At $N=22$ the excited state $\mathrm{MRCI}_{\mathrm{c}}$ descriptions cover about $2 \times 10^{6} \mathrm{SACs}$ for both spatial symmetries. For $\mathrm{MRCI}_{\mathrm{S}}$ the dimensions are seen to vary strongly among the excited states. At $N=22$ the $\mathrm{MRCI}_{\mathrm{s}}$ expansions cover $3 \%\left(1 \mathrm{~B}_{\mathrm{u}}^{+}\right)$, $35 \%\left(2 \mathrm{~A}_{\mathrm{g}}^{-}\right)$, and $41 \%\left(1 \mathrm{~B}_{\mathrm{u}}^{-}\right)$of the associated $\mathrm{MRCI}_{\mathrm{c}}$ basis sets, respectively. These percentages become systematically smaller with increasing polyene size $N$ showing that the computational advantage of $\mathrm{MRCI}_{\mathrm{s}}$ over $\mathrm{MRCI}_{\mathrm{c}}$ increases with $N$.

If one includes also $\sigma$-MOs into the active space, then the $\mathrm{MRCI}_{\mathrm{c}}$ dimensions become rapidly very large with increasing $N$. Therefore, we chose in these cases the more cost effective $\mathrm{MRCI}_{\mathrm{s}}$ approach. The sizes of the excited state reference sets $R(s, N)$ resulting from the same DCI density threshold $\theta=92 \%$, which was used for the ( $\pi$ ) active spaces of the 
TABLE III. OM2/MRCI $\mathrm{SAC}$ dimensions for the $\left(\sigma_{r}, \pi\right)$ and $(\sigma, \pi)$ active spaces.

\begin{tabular}{|c|c|c|c|c|c|c|c|c|}
\hline \multirow[b]{2}{*}{$N$} & \multicolumn{2}{|c|}{$1 \mathrm{~A}_{\mathrm{g}}^{-}$} & \multicolumn{2}{|c|}{$2 \mathrm{~A}_{\mathrm{g}}^{-}$} & \multicolumn{2}{|c|}{$1 \mathrm{~B}_{\mathrm{u}}^{-}$} & \multicolumn{2}{|c|}{$1 \mathrm{~B}_{\mathrm{u}}^{+}$} \\
\hline & $\left(\sigma_{r}, \pi\right)$ & $(\sigma, \pi)$ & $\left(\sigma_{r}, \pi\right)$ & $(\sigma, \pi)$ & $\left(\sigma_{r}, \pi\right)$ & $(\sigma, \pi)$ & $\left(\sigma_{r} \pi\right)$ & $(\sigma, \pi)$ \\
\hline 4 & 350 & 2234 & 3028 & 16468 & $\ldots$ & $\ldots$ & 1696 & 13646 \\
\hline 6 & 1263 & 9661 & 32419 & 284025 & 26840 & 242105 & 7623 & 63402 \\
\hline 8 & 3782 & 28254 & 143995 & 1665782 & 160503 & 1621807 & 22992 & 192416 \\
\hline 10 & 8557 & 65887 & 518699 & $t x$ & 703502 & $t x$ & 82611 & 715746 \\
\hline 12 & 16874 & 132542 & 1502330 & $t x$ & 2191414 & $t x$ & 260465 & 2291309 \\
\hline
\end{tabular}

PPP model, are listed in Table S5 in the SM for the complete $(\sigma, \pi)$ active spaces. One finds that the reference sets $R(s, N)$ of the covalent excitation states are essentially unchanged upon expanding the $(\pi)$ active spaces of PPP to the complete $(\sigma, \pi)$ active spaces of OM2. In contrast, the sets $R\left(1 \mathrm{~B}_{\mathrm{u}}^{+}, N\right)$ become considerably larger. Thus, the properties of the $1 \mathrm{~B}_{\mathrm{u}}^{+}$ state should be more strongly affected by $\sigma-\pi$ and $\sigma-\sigma$ correlations than those of the covalent excitations. Note that the strong influence of these correlations on the $1 \mathrm{~B}_{\mathrm{u}}^{+}$state has been first described for $N=4,6$ by Cave and Davidson. ${ }^{51,52}$

We used the reference sets $R(s, N)$ of $\mathrm{OM} 2 / \mathrm{MRCI}_{\mathrm{s}}(\sigma, \pi)$ determined from $\mathrm{OM} 2 / \mathrm{DCI}(\sigma, \pi)$ also for $\mathrm{OM}_{2} / \mathrm{MRCI}_{\mathrm{s}}(\pi)$ and $\mathrm{OM} 2 / \mathrm{MRCI}_{\mathrm{S}}\left(\sigma_{r}, \pi\right)$ to single out the effects of $\sigma-\pi$ and $\sigma-\sigma$ correlations. For all active spaces the OM2/MRCI ground state was represented by DCI.

Table III lists the SAC dimensions of the $\mathrm{OM} 2 / \mathrm{MRCI}_{\mathrm{S}}$ expansions constructed from the reference sets in Table S5 (SM) for the restricted $\left(\sigma_{r}, \pi\right)$ and the complete $(\sigma, \pi)$ active spaces. Note that for $N=10$ the $\operatorname{MRCI}_{\mathrm{S}}(\sigma, \pi)$ matrix of the $2 \mathrm{~A}_{\mathrm{g}}^{-}$and $1 \mathrm{~B}_{\mathrm{u}}^{-}$states did not fit anymore into the $64 \mathrm{GiB}$ of dynamic memory available on the computer used by us for these calculations, because the dimensions were 5030739 and 5819 320. Similarly, for $N=12$ the $\operatorname{MRCI}_{\mathrm{s}}(\sigma, \pi)$ matrix of the $1 \mathrm{~B}_{\mathrm{u}}^{+}$state exceeded the available memory. But whereas in the latter case the iterative diagonalization of the matrix through repeated reading of the matrix from hard disk could be completed within about half a day of computer time, each of the former diagonalizations would have required an estimated time of 40 days, which we considered as "too expensive" $(t x)$. Correspondingly, these omitted computations are labeled by " $t x$ " in Table III.

\section{Polyene geometries}

For comparison with PPP/MRCI $\mathrm{M}_{\mathrm{c}}$ we carried out $\mathrm{OM} 2 / \mathrm{MRCI}_{\mathrm{c}}(\pi)$ calculations at the polyene model geometries employed in PPP. ${ }^{7,11}$ This geometry featured $120^{\circ}$ bond angles within the carbon backbone and lengths of $1.45 \AA$ and $1.35 \AA$ for the $\mathrm{C}-\mathrm{C}$ single and $\mathrm{C}=\mathrm{C}$ double bonds, respectively. The $\mathrm{C}-\mathrm{H}$ bond lengths were set to $1 \AA$ and the $\mathrm{C}-\mathrm{C}-\mathrm{H}$ bond angles to $120^{\circ}$.

To study the effects of geometry relaxation on vertical excitation energies we subsequently calculated by OM2/DCI optimized geometries of the $1 \mathrm{~A}_{\mathrm{g}}^{-}$state for the $(\pi)$ and $(\sigma, \pi)$ active spaces. For $N=12$ we checked to what extent the addition of a second reference SAC, which was the $\mathrm{HOMO}^{2} \rightarrow \mathrm{LUMO}^{2}$ double excitation, to the reference set $R\left(1 \mathrm{~A}_{\mathrm{g}}^{-}, N\right)$ changes the OM2/MRCI $\mathrm{I}_{\mathrm{s}}$ optimized geometry (method MRCI*). During geometry optimizations the planar $C_{2 h}$ symmetry was strictly enforced.

\section{RESULTS}

Here we will first address the question, why the early $\mathrm{PPP} / \mathrm{MRCI}_{\mathrm{s}}$ descriptions of the electronic excitations in long polyenes were so successful despite the use of model geometries instead of optimized geometries and the inherent neglect of $\sigma-\pi$ interactions. To separate these two issues we first compare PPP/MRCI with $\mathrm{OM} 2 / \mathrm{MRCI}(\pi)$ for equivalent model geometries (cf. Sec. II D).

Such a comparison requires a MRCI method, which treats the PPP and OM2 models of the polyenes as similarly as possible. Here, the established state specific $\mathrm{MRCI}_{\mathrm{s}}$ approach $^{7,11}$ has the disadvantage of yielding different reference sets $R(s, N)$ and correspondingly different SAC dimensions for the excited states despite the use of an identical DCI density threshold $\theta . \mathrm{MRCI}_{\mathrm{c}}$ (Sec. II C), in contrast, provides identical SAC bases for PPP and OM2 $(\pi)$ and, therefore, allows us to single out the effects of the different Hamiltonians on calculated polyene spectra. Choosing $\mathrm{MRCI}_{\mathrm{c}}$ instead of $\mathrm{MRCI}_{\mathrm{s}}$ raises the initial question, how well PPP/MRCI $\mathrm{I}_{\mathrm{c}}$ compares with $\mathrm{PPP} / \mathrm{MRCI}_{\mathrm{s}}$.

\section{A. PPP/MRCl and $P P P / M R C l_{c}$ are nearly equivalent}

To check how well PPP/MRCI $\mathrm{S}_{\mathrm{S}}$ excitation energies $\Delta E_{\mathrm{s}}(s)$ of the $\pi$-electron excitations $s \in\left\{2 \mathrm{~A}_{\mathrm{g}}^{-}, 1 \mathrm{~B}_{\mathrm{u}}^{-}, 1 \mathrm{~B}_{\mathrm{u}}^{+}\right\}$ are reproduced by $\mathrm{PPP} / \mathrm{MRCI}_{\mathrm{c}}$ we applied both methods to the polyenes in the range $4 \leq N \leq 22$ (cf. Sec. II C, numerical values are listed in Tables S6 and S7 in the SM).

Figure 3 compares the excitation energies $\Delta E_{\mathrm{s}}(s, N)$ (gray) and $\Delta E_{\mathrm{c}}(s, N)$ (black) of three low-lying $\pi$-electron excitations obtained with $\mathrm{PPP} / \mathrm{MRCI}_{\mathrm{s}}$ and $\mathrm{PPP} / \mathrm{MRCI}_{\mathrm{c}}$, respectively. Because the ground state is described by DCI in both cases, differences reflect those of excited state energies $E(s, N)$. As a consequence of the variational principle, the energies $E_{\mathrm{s}}(s, N)$ resulting from the less extended $\mathrm{MRCI}_{\mathrm{s}}$ treatment are everywhere above the $\mathrm{MRCI}_{\mathrm{c}}$ energies $E_{\mathrm{c}}(s, N)$. Apparently, however, the differences $\Delta E_{\mathrm{s}-\mathrm{c}}(s, N) \equiv E_{\mathrm{s}}(s, N)$ $-E_{\mathrm{c}}(s, N)$ are small such that the two MRCI approaches represent similar descriptions.

The differences $\Delta E_{\mathrm{s}-\mathrm{c}}(s, N)$ are displayed in a magnified fashion in Figure S18 in the SM and are thoroughly discussed in the accompanying text. In particular, we discuss the fact 


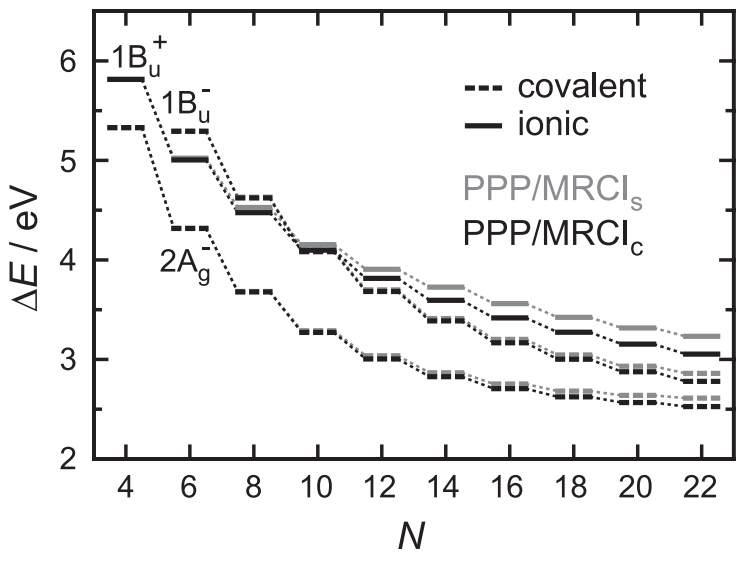

FIG. 3. PPP/MRCI $\left(\right.$ gray) and $\mathrm{PPP} / \mathrm{MRCI}_{\mathrm{c}}$ (black) excitation energies $\Delta E(s, N)$ of the singlet states $s \in\left\{2 \mathrm{~A}_{\mathrm{g}}^{-}, 1 \mathrm{~B}_{\mathrm{u}}^{-}, 1 \mathrm{~B}_{\mathrm{u}}^{+}\right\}$in the polyenes with $4 \leq N \leq 22 \pi$-electrons.

that $\mathrm{MRCI}_{\mathrm{S}}$ results are connected with a certain degree of uncertainty, because there are many different and a priori reasonable procedures for reference selection. However, no such uncertainty is connected with $\mathrm{MRCI}_{\mathrm{c}}$. For our comparison of PPP with OM2 we therefore chose the latter approach.

\section{B. PPP/MRCI ${ }_{\mathrm{C}}$ and $\mathrm{OM} 2 / \mathrm{MRCl}_{\mathrm{c}}(\pi)$ are nearly equivalent at the PPP model geometries}

If one chooses the $(\pi)$ active spaces in $\mathrm{OM} 2 / \mathrm{MRCI}_{\mathrm{c}}$ treatments, then the variational CI spaces have the same dimensions (see Table II) as in PPP/MRCI ${ }_{c}$. If one additionally chooses for OM2 regular model geometries, which are identical to those used in PPP (see Sec. II D), then $\mathrm{MRCI}_{\mathrm{c}}$ excitation energy differences are exclusively caused by differences of the two model Hamiltonians PPP and OM2.

A striking difference is the artificial PAS (Refs. 14, 19) of the PPP model, which is absent in OM2 (cf. Sec. I A). Correspondingly, the classification of a given $\mathrm{OM} 2 / \mathrm{MRCI}_{\mathrm{c}}(\pi)$ state as "+" or "-_" must rely on matchings with PPP/MRCI expansions. Fortunately these matchings are straightforward, because the PPP and OM2 CI expansions turned out to be very similar.

Consider, for instance, the PPP/MRCI $\mathrm{I}_{\mathrm{c}}$ and $\mathrm{OM} 2 / \mathrm{MRCI}_{\mathrm{c}}(\pi)$ wave functions of the $2 \mathrm{~A}_{\mathrm{g}}^{-}$state in hexatriene, whose most important contributions are depicted in Figs. 4(a) and 4(b), respectively. As a consequence of the PAS, the PPP/CI wave function displays a particle-hole symmetry, which is obtained by taking the mirror images of the MO energy levels and orbital occupation patterns with respect to the Fermi level and by subsequently exchanging particles (filled circles) with holes (empty circles) and vice versa. Hence, the shown double excitation is invariant under this operation, whereas the two single excitations map onto each other. Therefore, the coefficients of the latter SACs have identical absolute values but different signs. Here, the sign change indicates the "-" symmetry class.

The prerequisite for the PAS and the associated particlehole symmetry is that the particle and hole levels are symmetrically spaced around the Fermi level. Figure S19 in the SM demonstrates for hexatriene that this symmetry is present in

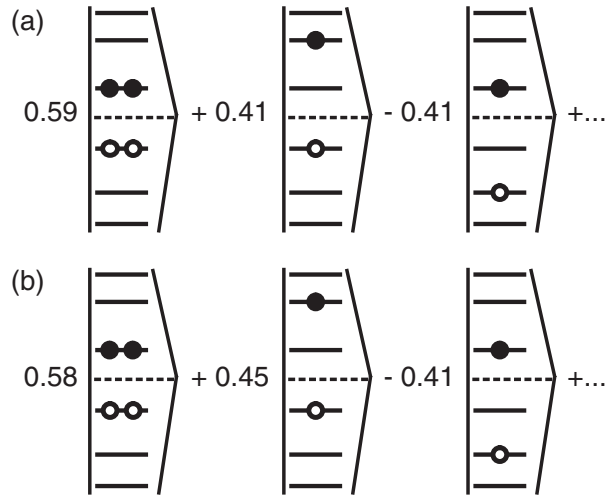

FIG. 4. Three singlet SACs strongly contributing to the $2 \mathrm{~A}_{\mathrm{g}}^{-}$excitation of hexatriene $(N=6)$ according to (a) PPP/MRCI $\mathrm{c}_{\mathrm{c}}$ and (b) $\mathrm{OM} 2 / \mathrm{MRCI}_{\mathrm{c}}(\pi)$ in a particle-hole representation covering the $(\pi)$ active space.

PPP but is violated in OM2. As a consequence and as shown in Fig. 4(b) the OM2/CI coefficients of two single excitations have slightly different absolute values and the OM2/CI wave function lacks the " + / -" symmetry. On the other hand, the absolute values of the PPP and OM2 CI coefficients are quite similar indicating that the PAS is only weakly broken in the transition from PPP to $\mathrm{OM} 2(\pi)$. As a result, the $\mathrm{OM} 2 / \mathrm{MRCI}_{\mathrm{c}}(\pi)$ wave functions are easily classified as "+" or "-" by matching onto their PPP/MRCI ${ }_{c}$ relatives. Therefore, we will maintain the PAS symmetry labels " + / -" also in denoting excited states calculated by OM2/MRCI.

The close match of the CI expansions lets expect that the size dependence of the polyene $\mathrm{MRCI}_{\mathrm{c}}$ excitation energies should be similar in OM2 and PPP. This expectation is verified by Fig. 5, which displays for five low-lying singlet excitations the differences $\Delta \Delta E_{H}$ of excitation energies calculated by the two methods at identical geometries. Because the ground state correlation energies resulting from PPP/MRCI ${ }_{c}$ and $\mathrm{OM} 2 / \mathrm{MRCI}_{\mathrm{c}}(\pi)$ are nearly identical (see Tables S7 and S8 in the SM), the shown differences $\Delta \Delta E_{H}$ are mainly due to different excited state energies (relative to the respective HF energies).

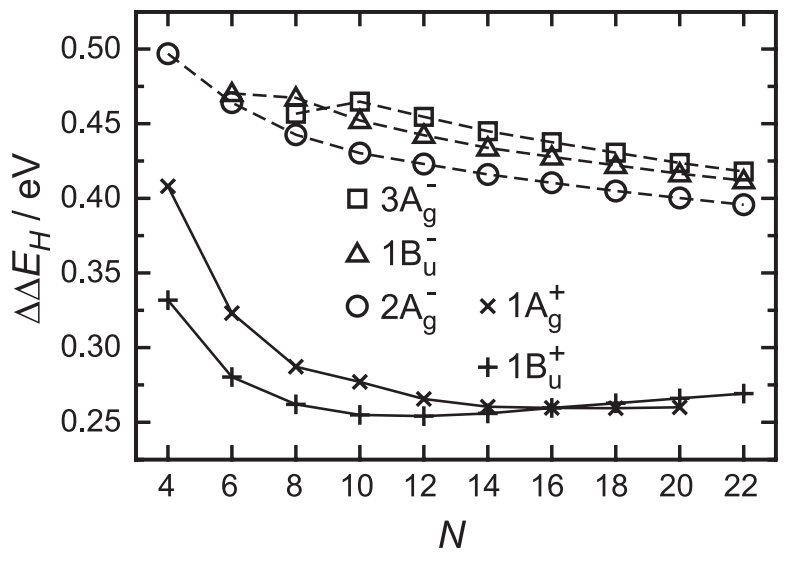

FIG. 5. Differences $\Delta \Delta E_{H}=\Delta E_{\mathrm{OM} 2}-\Delta E_{\mathrm{PPP}}$ of excitation energies obtained with PPP and OM2 by $\operatorname{MRCI}_{\mathrm{c}}(\pi)$ for the singlet excitations $s \in\left\{2 \mathrm{~A}_{\mathrm{g}}^{-}, 1 \mathrm{~B}_{\mathrm{u}}^{+}, 1 \mathrm{~B}_{\mathrm{u}}^{-}, 3 \mathrm{~A}_{\mathrm{g}}^{-}, 1 \mathrm{~A}_{\mathrm{g}}^{+}\right\}$of polyenes with regularly alternating model geometries and sizes $N$. 
In the longer polyenes $(N \geq 8) \mathrm{MRCI}_{\mathrm{c}}(\pi)$ predicts for the two ionic states $1 \mathrm{~B}_{\mathrm{u}}^{+}$and $1 \mathrm{~A}_{\mathrm{g}}^{+}$excitation energies which are by about $0.27 \mathrm{eV}$ larger for OM2 than for PPP. For the three covalent states $2 \mathrm{~A}_{\mathrm{g}}^{-}, 1 \mathrm{~B}_{\mathrm{u}}^{-}$, and $3 \mathrm{~A}_{\mathrm{g}}^{-}$, still larger blue shifts are obtained. They are comparable for all three excitations, decrease a little with $N$, but still measure at least $0.40 \mathrm{eV}$ at $N=22$.

Thus, if one would shift the PPP/MRCI ${ }_{c}$ excitation energies of the $1 \mathrm{~B}_{\mathrm{u}}^{+}$state in Fig. 3(a), by $0.27 \mathrm{eV}$ and those of the covalent $2 \mathrm{~A}_{\mathrm{g}}^{-}$and $1 \mathrm{~B}_{\mathrm{u}}^{-}$excitations by about $0.43 \mathrm{eV}$ toward larger values, then one would get a visualization of the $\mathrm{OM} 2 / \mathrm{MRCI}_{\mathrm{c}}(\pi)$ prediction on the excitation energies of these three states for regular geometries. One concludes, in particular, that $\mathrm{OM} 2 / \mathrm{MRCI}_{\mathrm{c}}(\pi)$ assigns smaller values to the energy gaps $\Delta E_{\mathrm{BA}}$ than $\mathrm{PPP} / \mathrm{MRCI}_{\mathrm{c}}$. At $N=20$, for instance, the gap is predicted at $0.45 \mathrm{eV}(\mathrm{OM} 2)$ and $0.58 \mathrm{eV}(\mathrm{PPP})$, respectively. However, apart from these nearly uniform spectral shifts of the covalent and ionic excitations, respectively, $\mathrm{PPP} / \mathrm{MRCI}_{\mathrm{c}}$ and $\mathrm{OM} 2 / \mathrm{MRCI}_{\mathrm{c}}(\pi)$ yield essentially equivalent descriptions of the $\pi$-electron excitations in regularly alternating polyenes.

\section{OM2 optimized geometries}

As one can conclude from the $\pi$-electron bond orders resulting from PPP/CI descriptions of polyene ground states, the bond length alternation should vary within a given molecule. The alternation should be pronounced near the two ends of the chain and reduced at its center. ${ }^{7}$ Unfortunately, $\pi$-electron models such as PPP do not enable a rigorous optimization of the polyene geometries. This drawback is removed with OM2, because it includes all valence electrons. Here, the question arises, which level of theory one should choose for the computation of ground state geometries. This question is important, because calculated vertical excitation energies will differentially and sensitively depend on the bond length alternation in the chosen ground state geometry ${ }^{7,18,45}$ (cf. also Sec. III F in the SM).

As a simple observable for judging the bond length alternation we chose the order parameter ${ }^{7}$

$$
\Delta l_{m}=(-1)^{m}\left(l_{m}-\bar{l}_{c}\right), \quad m=1,2, \ldots, N-1,
$$

where $m$ counts the $\mathrm{C}-\mathrm{C}$ bonds along the polyene chain, $c \equiv N / 2$ is the number of the central bond, and $\bar{l}_{c} \equiv\left(l_{c}\right.$ $\left.+l_{c+1}\right) / 2$ is the average length of the central $\mathrm{C}-\mathrm{C}$ bonds. In a regularly alternating polyene the order parameter $\Delta l_{m}$ is constant and positive along the chain. For realistic polyene geometries $\Delta l_{m}$ will vary along the chain. Here, large values of $\Delta l_{m}$ indicate a pronounced and smaller values indicate a reduced alternation at bond $m$.

To understand the geometrical effects of $\pi$-electron correlation and of $\sigma-\pi$ and $\sigma-\sigma$ interactions we have calculated the $C_{2 \mathrm{~h}}$ ground state geometry of dodecahexaene at different levels of theory. We applied $\mathrm{OM} 2 / \mathrm{HF}, \mathrm{OM} 2 / \mathrm{DCI}(\pi)$, $\mathrm{OM} 2 / \mathrm{DCI}(\sigma, \pi)$, and $\mathrm{OM} 2 / \mathrm{MRCI}^{*}(\sigma, \pi)$ (which includes two references, cf. Sec. II D). Figure 6 compares the order parameters $\Delta l_{m}$ obtained by these methods for the bonds $m=6,7, \ldots, 11$, i.e., $m-6 \geq 0$.

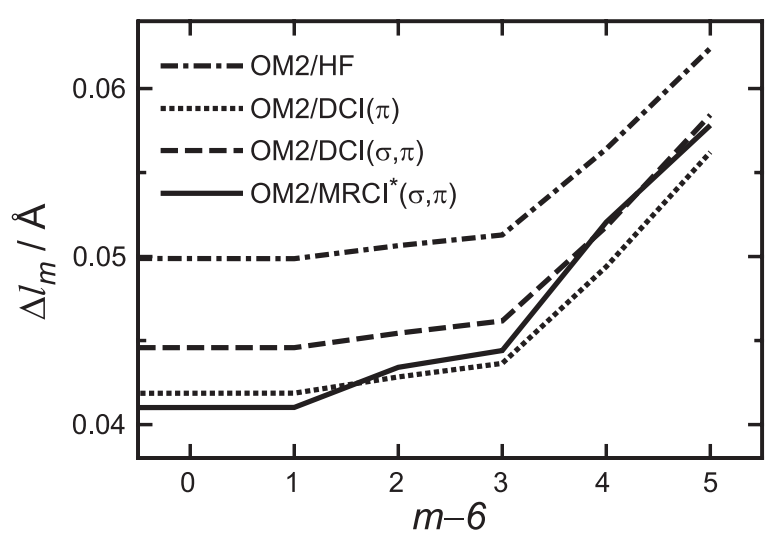

FIG. 6. Order parameter $\Delta l_{m}$ (Eq. (1)) evaluated for the ground state of dodecahexaene $(N=12)$ using different levels of theory (the parameters $\bar{l}_{6}$ are listed in Table S9 in the SM).

Figure 6 reveals that the bond alternation $\Delta l_{m}$ is largest at the $\mathrm{OM} 2 / \mathrm{HF}$ ground state geometry (dashed-dotted curve). It decreases from the ends of the polyene chain toward the central bond $m-6=0$. Including $\pi$-electron correlation at the DCI level decreases the alternation $\Delta l_{m}$ (dotted curve). Adding $\sigma-\pi$ and $\sigma-\sigma$ interactions at the $\operatorname{OM} 2 / \operatorname{DCI}(\sigma, \pi)$ level slightly increases $\Delta l_{m}$ again (dashed curve). Extending the CI treatment beyond that level $\left[\mathrm{OM} 2 / \mathrm{MRCI}^{*}(\sigma, \pi)\right]$ reverses this increase (solid curve). Thus, the inclusion of triply and quadruply excited SACs into the CI basis significantly changes the ground state geometry although it decreases the ground state energy by only $0.029 \mathrm{eV}$.

As a result, the much less costly $\mathrm{OM} 2 / \mathrm{DCI}(\pi)$ calculation yields an alternation, which is very close to that of the extended OM2/MRCI* $(\sigma, \pi)$ treatment (the six non-redundant $\mathrm{C}-\mathrm{C}$ bond lengths $l_{m}$ show a root mean square deviation of only $0.002 \AA$ ). Therefore, we chose the OM $2 / \mathrm{DCI}(\pi)$ optimized geometries for all further calculations.

The OM2/DCI $(\pi)$ geometries are given in the SM (Table S17). In the SM, Figure S20 and Table S11 additionally compare the alternations $\Delta l_{m}$ for $N=10,20,30$, and 40 . $\Delta l_{m}$ is shown to become nearly constant near the chain centers at large $N$. Here, the geometry is quite close to experimental finding $\mathrm{s}^{53}$ for the infinite chain (cf. SM) such that $\mathrm{OM} 2 / \mathrm{DCI}(\pi)$ geometries are reasonable at large $N$. For $N=4$ the $\mathrm{OM} 2 / \operatorname{DCI}(\pi)$ bond lengths differ from the experimental values ${ }^{54}$ by a root mean square deviation of $0.007 \AA$. Thus, the $\mathrm{OM} 2 / \mathrm{DCI}(\pi)$ polyene geometries should be reasonable for all $N$.

\section{Spectral effects of geometry optimization}

With the $\mathrm{OM} 2 / \mathrm{DCI}(\pi)$ geometries now at hand we can address the question how geometry optimization changes the $\mathrm{OM} 2 / \mathrm{MRCI}_{\mathrm{c}}(\pi)$ excitation energies. The answer is given by Fig. 7, which shows the effect $\Delta \Delta E_{G}$ of geometry optimization on the singlet excitation energies in the polyenes with $4 \leq N \leq 22 \pi$-electrons. $\Delta \Delta E_{G}$ are the differences between the excitation energies $\Delta E_{\text {optimized }}$ and $\Delta E_{\text {regular }}$ at the optimized and regular polyene geometries, respectively. 


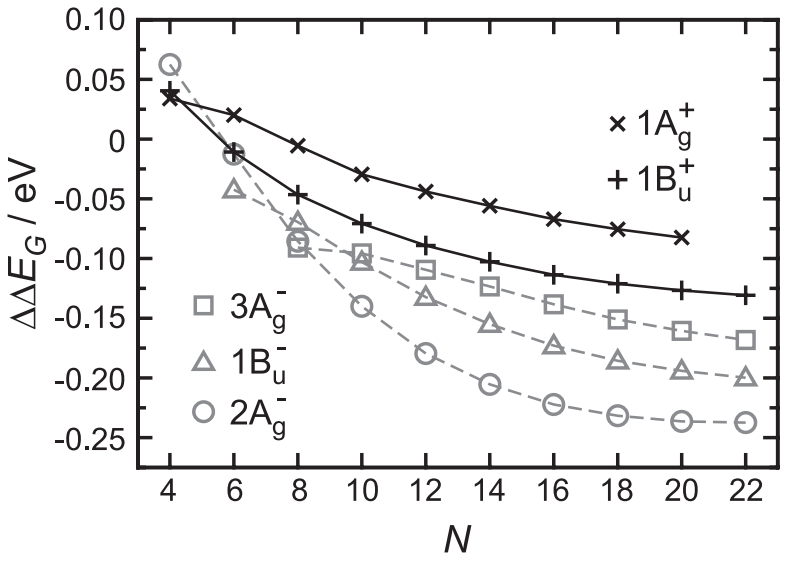

FIG. 7. Changes $\Delta \Delta E_{G}=\Delta E_{\text {optimized }}-\Delta E_{\text {regular }}$ of vertical $\mathrm{OM} 2 / \mathrm{MRCI}_{\mathrm{c}}(\pi)$ excitation energies $\Delta E$, which are caused by $\mathrm{OM} 2 / \mathrm{DCI}(\pi)$ ground state geometry optimization.

In the polyenes with $N \geq 8$ all excitation energy differences $\Delta \Delta E_{G}$ are seen to be negative for all five excited states, i.e., the transition from a constant alternation $\Delta l_{m}^{\mathrm{PPP}}=0.05 \AA$ to more realistic geometries generally leads to lower excitation energies. According to Fig. 7 this lowering is larger for the covalent (gray symbols) than for the ionic states (black symbols).

The geometry-induced excitation energy lowering $\Delta \Delta E_{G}$ partially compensates the excitation energy increase $\Delta \Delta E_{H}$ documented in Fig. 5, which results from abandoning PPP in favor of OM2 (while maintaining the restriction of dynamic correlations to the $\pi$-electrons). Thus, polyene singlet spectra calculated by $\mathrm{OM} 2 / \mathrm{MRCI}_{\mathrm{c}}(\pi)$ at realistic geometries more closely resemble the PPP/MRCI predictions than originally suggested by Fig. 5 .

The fact that the covalent states experience for $N \geq 8$ larger redshifts $\Delta \Delta E_{G}$ than the ionic states upon geometry optimization is explained by the early PPP/MRCI calculations. ${ }^{7}$ Accordingly, a removal of the alternation $\left(\Delta l_{m}\right.$ $=0$ ) reduces the covalent excitation energies substantially (removing, in particular, the covalent gap at $N \rightarrow \infty$ ), while it decreases the ionic excitation energies much less (preserving, in particular, a sizable optical gap at $N \rightarrow \infty$ ). Reconsidering Fig. 6 (and Fig. S20 in the SM) shows that $\Delta l_{m}$ is smaller than the standard PPP value $\Delta l_{m}^{\mathrm{PPP}}=0.05 \AA$ in most parts of longer polyenes (except at the ends) after geometry optimization. The reduced $\Delta l_{m}$ thus rationalize the red-shifts $\Delta \Delta E_{G}$ shown in Fig. 7. In the shortest polyene $(N=4)$, which exclusively consists of "ends," the alternation is $0.055 \AA$ and, thus, larger than $\Delta l_{m}^{\mathrm{PPP}}$. Correspondingly, in Fig. 7 the values $\Delta \Delta E_{G}>0$ at $N=4$ signify blue shifts of all excitation energies.

As a result, the general trends of the excitation energy shifts $\Delta \Delta E_{G}$ in Fig. 7 follow from different alternations in the optimized and regular geometries. The details of these effects, however, which indicate that, in each of the two PAS classes of polyene excitations, the red-shifts $\Delta \Delta E_{G}(s)$ become smaller with increasing excitation energy $\Delta E(s)$ of state $s$, are a surprise.

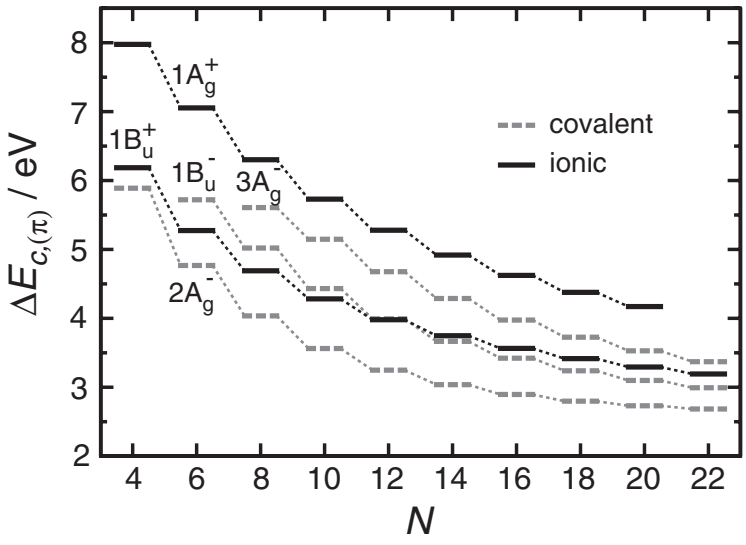

FIG. 8. Vertical OM2/MRCI $\mathrm{C}_{\mathrm{c}}(\pi)$ excitation energies $\Delta E_{\mathrm{c},(\pi)}$ evaluated at the $\mathrm{OM} 2 / \mathrm{DCI}(\pi)$ optimized ground state geometries as a function of the polyene length $N$.

For five low-lying $\pi$-electron excitations $s$, Fig. 8 displays the vertical transition energies $\Delta E_{\mathrm{c},(\pi)}(s, N)$ predicted by $\mathrm{OM} 2 / \mathrm{MRCI}_{\mathrm{c}}(\pi)$ at the optimized ground state geometries as a function of $N$. The shown $N$-dependence closely resembles that of the early PPP/MRCI descriptions $^{11}$ (Fig. 2). However, comparing Figs. 2 and 8 reveals two marked differences. First, the new calculations cover a larger range of polyene sizes. Second, according to $\mathrm{OM} 2 / \mathrm{MRCI}_{\mathrm{c}}(\pi)$ the $1 \mathrm{~B}_{\mathrm{u}}^{-}$ state appears below the $1 \mathrm{~B}_{\mathrm{u}}^{+}$state near $N=14$, whereas early PPP/MRCI ${ }_{\mathrm{s}}$ (like the new PPP/MRCI ${ }_{\mathrm{c}}$, Fig. 3(a)) localized this level switch at $N=10$.

\section{E. Transition dipole moments of the $1 \mathrm{~B}_{\mathrm{u}}^{-}$state}

As discussed in Sec. III B the PAS is only weakly broken in OM2. Therefore, the $1 \mathrm{~A}_{\mathrm{g}}^{-} \rightarrow 1 \mathrm{~B}_{\mathrm{u}}^{-}$transitions carry non-vanishing but very small transition dipole moments. Figure S22 and Sec. III G in the SM present and discuss corresponding $\mathrm{OM} 2 / \mathrm{MRCI}_{\mathrm{c}}(\pi)$ results. Accordingly, the squared transition dipole moments $d^{2}\left(1 \mathrm{~B}_{\mathrm{u}}^{+}\right)$are on an average by a factor of $4 \times 10^{4}$ larger than $d^{2}\left(1 \mathrm{~B}_{\mathrm{u}}^{-}\right)(6 \leq N \leq 22)$ with the exception of $N=12$, where the quasi-degeneracy of the two $\mathrm{B}_{\mathrm{u}}$ states enhances $d^{2}\left(1 \mathrm{~B}_{\mathrm{u}}^{-}\right)$by a factor of $10^{2}$.

The quoted factors are much larger than corresponding ratios of oscillator strengths published by other authors. Zhang and $\mathrm{Liu}^{27}$ derive for the polyenes with $6 \leq N \leq 12$ from non-PAS-PPP/full-CI and $a b$ initio CI-singles calculations factors of 30 and from time dependent density functional theory factors of $4 \times 10^{3}$. Nakayama et al. ${ }^{25}$ obtained from perturbation theory combined with CASSCF factors of $2 \times 10^{2}$ $(N=6,8)$. As a result it seems that in OM2 the breaking of the PAS is much less pronounced than in other approaches.

\section{F. Effects of $\sigma-\pi$ and $\sigma-\sigma$ correlation}

Up to now we have solely considered dynamical correlations among $\pi$-electrons. However, the valence shell model OM2 additionally enables a study of $\sigma-\pi$ and $\sigma-\sigma$ correlations. For this purpose we have introduced in Sec. II B the CI active spaces $(\sigma, \pi)$ and $\left(\sigma_{r}, \pi\right)$. As explained in Sec. II C the 


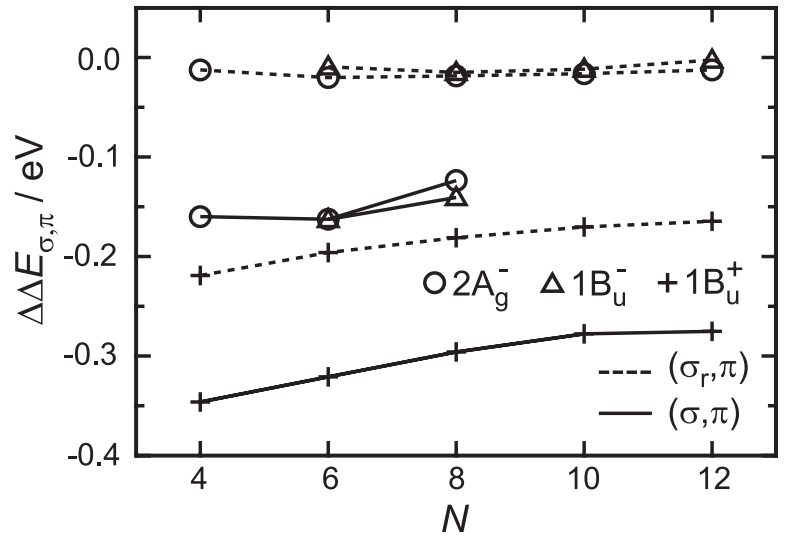

FIG. 9. Effects $\Delta \Delta E_{\sigma, \pi}$ of $\sigma-\pi$ and $\sigma-\sigma$ correlations on the vertical transition energies $\Delta E_{\mathrm{S}}(s, N)$ of the singlet excitations $s \in\left\{1 \mathrm{~B}_{\mathrm{u}}^{+}, 2 \mathrm{~A}_{\mathrm{g}}^{-}, 1 \mathrm{~B}_{\mathrm{u}}^{-}\right\}$as functions of $N$. The changes $\Delta \Delta E_{\sigma, \pi}(s, N)$ of the $\mathrm{OM} 2 / \mathrm{MRCI}_{\mathrm{s}}(\pi)$ excitation energies $\Delta E_{\mathrm{S},(\pi)}(s, N)$ upon including the restricted $\left(\sigma_{r}, \pi\right)$ and complete $(\sigma, \pi)$ active spaces into $\mathrm{OM} 2 / \mathrm{MRCI}_{\mathrm{s}}$ treatments are drawn as dashed and solid curves, respectively.

choice of these more extended active spaces leads to much larger variational spaces even in the cost-effective $\mathrm{MRCI}_{\mathrm{s}}$ setting (cf. Table III). Given the computational resources accessible to us this choice restricts the $\mathrm{MRCI}_{\mathrm{S}}$ calculation of the low-lying covalent excitations $2 \mathrm{~A}_{\mathrm{g}}^{-}$and $1 \mathrm{~B}_{\mathrm{u}}^{-}$to polyenes covering at most $N=8$ and $N=12$ carbon atoms, respectively.

For the three prominent singlet excitations, Fig. 9 shows the changes $\Delta \Delta E_{\sigma, \pi}(s, N)$ of the excitation energies $\Delta E_{\mathrm{s}}(s, N)$, which result from expanding the $(\pi)$ active space to the restricted $\left(\sigma_{r}, \pi\right)$ (dashed curves) and complete $(\sigma, \pi)$ (solid curves) active spaces. Note that we used the reference sets $R(s, N)$, which were determined for the $(\sigma, \pi)$ active spaces (cf. Table S5 in the SM) also for the $\operatorname{MRCI}_{\mathrm{s}}(\pi)$ and $\mathrm{MRCI}_{\mathrm{s}}\left(\sigma_{r}, \pi\right)$ calculations, because this procedure isolates the effects $\Delta \Delta E_{\sigma, \pi}(s, N)$ of dynamic $\sigma-\pi$ and $\sigma-\sigma$ correlations.

Figure 9 demonstrates that the transition energies of the $2 \mathrm{~A}_{\mathrm{g}}^{-}$and $1 \mathrm{~B}_{\mathrm{u}}^{-}$states are nearly invariant as long as only the restricted set of $\sigma$-MOs is added to the $(\pi)$ active space. If the remaining $2 N \sigma$-MOs are also included, their excitation energies decrease by about $0.15 \mathrm{eV}$. Whether this decrease becomes actually smaller for polyenes with $N \geq 8$ cannot be judged from our limited data basis. We therefore will assume that $\Delta \Delta E_{\sigma, \pi}(s, N) \approx 0.15 \mathrm{eV}$ for all $N$ and $s \in\left\{2 \mathrm{~A}_{\mathrm{g}}^{-}, 1 \mathrm{~B}_{\mathrm{u}}^{-}\right\}$.

In contrast, the excitation energies of the ionic $1 \mathrm{~B}_{\mathrm{u}}^{+}$ state are seen to be affected already by the extension to $\left(\sigma_{r}, \pi\right)$ and these redshifts are even larger than those experienced by the covalent excitations upon further extension to $(\sigma, \pi)$. Physically this difference points to the fact that the $1 \mathrm{~B}_{\mathrm{u}}^{+}$states consist of electron-hole pairs, which strongly disturb the charge distributions of the $\sigma$-cores (cf. Cave and Davidson $^{52}$ for the importance of $\sigma-\pi$ correlations in the $1 \mathrm{~B}_{\mathrm{u}}^{+}$-state of hexatriene). In the covalent excitations, in contrast, the $\pi$-electrons stay essentially at their ground state locations where they solely feature different spin coupling patterns and correspondingly weakened $\pi$-bonds. The redshifts $\Delta \Delta E_{\sigma, \pi}\left(1 \mathrm{~B}_{\mathrm{u}}^{+}, N\right)$ of $\Delta E_{\mathrm{s}}\left(1 \mathrm{~B}_{\mathrm{u}}^{+}, N\right)$ increase by about a factor of two, if all $\sigma-\pi$ and $\sigma-\sigma$ correlations are considered, and become smaller with increasing $N$. When plotted on a $1 /(N$ $+1)$ scale $\Delta \Delta E_{\sigma, \pi}\left(1 \mathrm{~B}_{\mathrm{u}}^{+}, N\right)$ approximately forms a straight line (data not shown). Therefore, one can easily extrapolate $\Delta \Delta E_{\sigma, \pi}\left(1 \mathrm{~B}_{\mathrm{u}}^{+}, N\right)$ to larger $N$ with the limiting value of $0.2 \mathrm{eV}$ at $N \rightarrow \infty$.

Note here that the vanishing or small values $\Delta \Delta E_{\sigma, \pi}(s, N)$ calculated for the covalent excitations hide the large influence, which the extension of the active space exerts on the energies of these states. OM2/DCI $(\pi)$ yields for the $1 \mathrm{~A}_{\mathrm{g}}^{-}$ground state, for instance, a correlation energy per ethylenic unit of $-0.26 \mathrm{eV}$, extension to $\left(\sigma_{r}, \pi\right)$ renders $-0.33 \mathrm{eV}$ and to $(\sigma, \pi)$ $-0.61 \mathrm{eV}$. The stated near invariance of the excitation energies in the transition $(\pi) \rightarrow\left(\sigma_{r}, \pi\right)$ thus demonstrates that the $\left(\sigma_{r}, \pi\right)$ correlations are extremely similar in all covalent states. Although the correlation energies of the covalent excitations slightly exceed that of the ground state in the $\left(\sigma_{r}, \pi\right) \rightarrow(\sigma, \pi)$ transition, the excited state correlations still largely resemble those in the ground state.

As we have seen above, including the complete $(\sigma, \pi)$ space shifts the transition energies of all three low-lying $\pi$ electron excitations by $0.15-0.3 \mathrm{eV}$ to the red and this effect is more pronounced for the $1 \mathrm{~B}_{\mathrm{u}}^{+}$state than for the $2 \mathrm{~A}_{\mathrm{g}}^{-}$and $1 \mathrm{~B}_{\mathrm{u}}^{-}$ states. Computational limitations have restricted the analysis for the $(\sigma, \pi)$ space and the covalent excitations to $N \leq 8$.

On the other hand, the available results provide reasonable estimates on the effects $\Delta \Delta E_{\sigma, \pi}(s, N)$ of the $(\sigma, \pi)$ correlations also for much larger polyenes. When adding these estimates to the $\mathrm{OM} 2 / \mathrm{MRCI}_{\mathrm{c}}(\pi)$ excitation energies $\Delta E_{\mathrm{c},(\pi)}(s, N)$ (cf. Fig. 8) one arrives at "best estimates" $\Delta E_{\mathrm{c},(\sigma, \pi)}(s, N)$ for the $\mathrm{OM} 2 / \mathrm{MRCI}_{\mathrm{c}}(\sigma, \pi)$ vertical transitions of the three prominent $\pi$-electron excitations $s \in\left\{2 \mathrm{~A}_{\mathrm{g}}^{-}, 1 \mathrm{~B}_{\mathrm{u}}^{+}, 1 \mathrm{~B}_{\mathrm{u}}^{-}\right\}$. The details of this construction are explained in Sec. IV in the SM. The resulting numerical values are listed in Table S14.

\section{DISCUSSION}

Figure 10(a) compares the thus obtained best estimates for the OM2/MRCI $(\sigma, \pi)$ vertical transitions to the $2 \mathrm{~A}_{\mathrm{g}}^{-}$and $1 \mathrm{~B}_{\mathrm{u}}^{+}$singlet excited states with our best estimate for the corresponding "experimental" gas phase data collected in Table S4 and explained in Sec. I B in the SM. The figure additionally contains the $\mathrm{OM} 2 / \mathrm{MRCI}_{\mathrm{c}}(\sigma, \pi)$ predictions on the vertical transitions to the $1 \mathrm{~B}_{\mathrm{u}}^{-}$state, for which no experimental data are available.

According to Fig. 10(a) our best estimate on the $\mathrm{OM} 2 / \mathrm{MRCI}_{\mathrm{c}}(\sigma, \pi)$ description of the $1 \mathrm{~B}_{\mathrm{u}}^{+}$excitation energies (black solid levels) matches the depicted experimental data (gray solid levels) state quantitatively very well as is apparent from the root mean square deviation (RMSD) of only $0.07 \mathrm{eV}$. Only at $N=4$ and $N \geq 12$ the calculated values $\Delta E_{\mathrm{c},(\sigma, \pi)}\left(1 \mathrm{~B}_{\mathrm{u}}^{+}, N\right)$ represent slight underestimates of the experimental vertical transitions. Also for the $2 \mathrm{~A}_{\mathrm{g}}^{-}$ state the experimental excitation energies are very well reproduced as long as $N<12$. For $N \geq 12$ the calculated values $\Delta E_{\mathrm{c},(\sigma, \pi)}\left(2 \mathrm{~A}_{\mathrm{g}}^{-}, N\right)$ increasingly overestimate the observed excitation energies. According to the $\mathrm{OM} 2 / \mathrm{MRCI}_{\mathrm{c}}(\pi)$ description shown in Fig. 8 the $1 \mathrm{~B}_{\mathrm{u}}^{-}$state becomes the first excited singlet state already for polyenes with $N>12$. In mild 


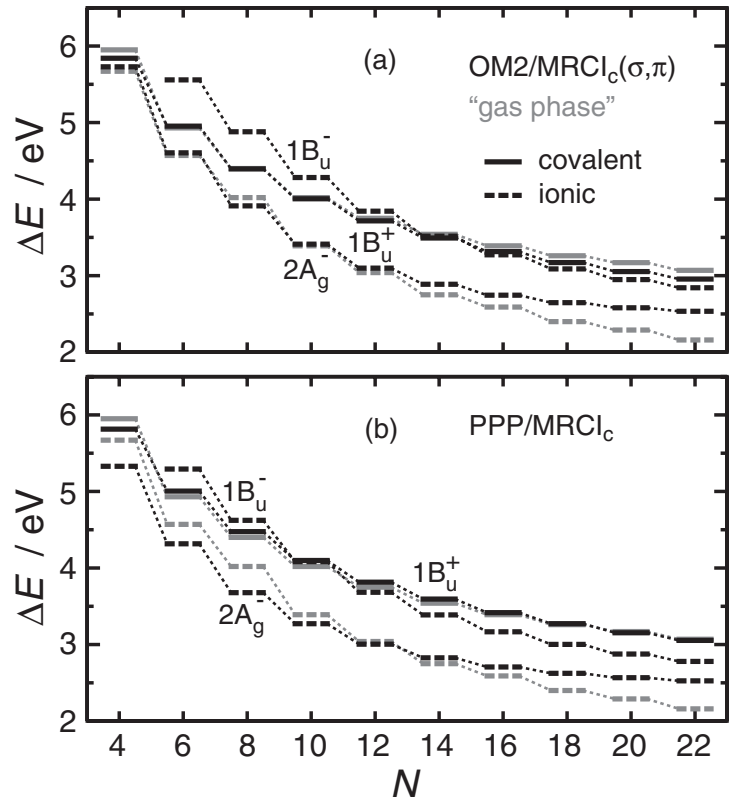

FIG. 10. (a) Best estimates for the vertical $\mathrm{OM} 2 / \mathrm{MRCI}_{\mathrm{c}}(\sigma, \pi)$ excitation energies $\Delta E_{\mathrm{c},(\sigma, \pi)}(s, N)$ (black) of the excited singlet states $s \in\left\{2 \mathrm{~A}_{\mathrm{g}}^{-}, 1 \mathrm{~B}_{\mathrm{u}}^{+}, 1 \mathrm{~B}_{\mathrm{u}}^{-}\right\}$in the polyenes with $4 \leq N \leq 22$ are compared with best estimates for the experimental gas phase values (gray) compiled by us for $s \in\left\{2 \mathrm{~A}_{\mathrm{g}}^{-}, 1 \mathrm{~B}_{\mathrm{u}}^{+}\right\}$(see Secs. I B and IV in the SM). (b) Corresponding comparison for $\mathrm{PPP} / \mathrm{MRCI}_{\mathrm{c}}$.

contrast, the best estimate in Fig. 10(a) assigns this level switch to polyenes with $N>14$.

The PPP/MRCI $I_{c}$ description of the $\pi-\pi^{*}$ energy levels shown in Fig. 10(b) does not differ much from $\mathrm{OM} 2 / \mathrm{MRCI}_{\mathrm{c}}(\sigma, \pi)$ as far as the $1 \mathrm{~B}_{\mathrm{u}}^{+}$excitation is concerned and also shows a RMSD of only $0.07 \mathrm{eV}$ from the experimental data. The $2 \mathrm{~A}_{\mathrm{g}}^{-}$state, however, is described at a comparable quality only for $N \geq 12$, where both descriptions overestimate the excitation energies. PPP/MRCI $\mathrm{I}_{\mathrm{c}}$ somewhat underestimates the $2 \mathrm{~A}_{\mathrm{g}}^{-}$excitation energies for $N<12$ quite in contrast to $\mathrm{OM} 2 / \mathrm{MRCI}_{\mathrm{c}}(\sigma, \pi)$ which, here, matches the experimental data much better.

A glance at Fig. 5, which illustrates the effects of including the $\sigma$-electrons by choosing OM 2 instead of PPP, explains the improved description of the $2 \mathrm{~A}_{\mathrm{g}}^{-}$excitation achieved with $\mathrm{OM} 2 / \mathrm{MRCI}_{\mathrm{c}}(\sigma, \pi)$ for $N<12$. This figure reveals a strong blue shift of the covalent excitations due to the transition from PPP to OM2 which, according to Fig. 7, remains for $N<12$ nearly unaffected by choosing optimized instead of regular geometries.

As a first result we thus may state that the transition from PPP to OM2, the inclusion of $\sigma-\pi$ and $\sigma-\sigma$ correlations, and the choice of optimized geometries yield quantitatively improved descriptions of the low-lying $\pi-\pi *$ excitations in the shorter polyenes $(N<12)$. Independently of the Hamiltonian, the $1 \mathrm{~B}_{\mathrm{u}}^{+}$excitation energies appear to be reasonable for $N>12$, whereas, here, the $2 \mathrm{~A}_{\mathrm{g}}^{-}$excitation energies deteriorate.

We will argue now that the systematic $\mathrm{MRCI}_{\mathrm{c}}$ overestimate of the $2 \mathrm{~A}_{\mathrm{g}}^{-}$excitation energies for $N>12$ is caused by an imbalanced description of ground and excited state correlations. This imbalance is shown to be due to the fact that the reference SACs employed for the covalent excited states do not cover necessary triples and quadruples. For the polyenes with $N>12$ this omission leads to increasingly poor estimates of the correlation energies in the covalent excited states and to a lacking size consistency of the associated excitation energies.

\section{A. Effects of imbalance}

We have introduced $\mathrm{MRCI}_{\mathrm{c}}$ as a more costly variant of the $\mathrm{MRCI}_{\mathrm{s}}$. The latter approach features for each excited state $s$ an individual selection of the sets $R(s, N)$ of reference SACs from the DCI wave function using a common density threshold. $\mathrm{MRCI}_{\mathrm{c}}$, in contrast, considers all singles and doubles as references such that it becomes a QCI treatment for all excited states. Both MRCI approaches choose the closed shell HF determinant as the sole reference for the ground state and, therefore, treat the ground state correlations at the DCI level. Thus, the two MRCI methods rest on the common assumption that the most important contributions to the wave functions of the excited states $s$ are collections $R(s, N)$ of singly and doubly excited SACs and that, concurrently, the HF determinant provides a reasonable and equally good zeroth order description of the ground state.

The $\mathrm{OM} 2 / \mathrm{QCI}(\pi)$ wave functions resulting from $\mathrm{OM} 2 / \mathrm{MRCI}_{\mathrm{c}}(\pi)$ now allow us to check whether the above assumptions are actually valid. Here we take advantage of the fact that the state local OM2/MRCI $(\pi)$ reference selection procedure, which applies a $92 \%$ density threshold at the DCI level, yields certain numbers $|R(s, N)|$ of singly and doubly excited reference SACs (see Table S15 in the SM). If one now collects the $|R(s, N)|$ most important contributions to the $\mathrm{QCI}(\pi)$ wave functions, one can find out whether these SACs

(i) cover similar percentages $T(s, N)$ of the $\mathrm{QCI}(\pi)$ wave functions for each state $s$ at a given $N$ and

(ii) solely comprise vanishing percentages $M(s, N)$ of triples and quadruples.

If both checks find positive answers, both MRCI methods should yield balanced treatments of the correlations in all states and, therefore, size-consistent excitation energies. Conversely, violations of the two requirements will point to certain imbalances. Note that this consistency check is close in spirit to the MRCI selection procedures used in Ref. 10.

To check (i) and (ii) we calculated the percentages $T(s, N)$ and $M(s, N)$ for the states $s \in\left\{1 \mathrm{~A}_{\mathrm{g}}^{-}, 2 \mathrm{~A}_{\mathrm{g}}^{-}, 1 \mathrm{~B}_{\mathrm{u}}^{-}, 1 \mathrm{~B}_{\mathrm{u}}^{+}\right\}$from the $\mathrm{OM} 2 / \mathrm{QCI}(\pi)$ wave functions. Figures 11 and 12 , respectively, display the corresponding results.

According to Fig. 11 the $|R(s, N)|$ most important SACs contribute for each polyene $N$ about equal percentages $T(s, N)$ to the $\mathrm{OM} 2 / \mathrm{QCI}(\pi)$ wave functions of the two covalent excitations $2 \mathrm{~A}_{\mathrm{g}}^{-}$and $1 \mathrm{~B}_{\mathrm{u}}^{-}$. The much smaller sets of important SACs characterizing the $1 \mathrm{~B}_{\mathrm{u}}^{+}$states nevertheless cover slightly larger percentages of the corresponding $\mathrm{OM} 2 / \mathrm{QCI}(\pi)$ wave functions. Considered as functions of $N$ the curves $T(s, N)$ are approximately parallel for all three excited states. For the ground state, in contrast, one notices that $T\left(1 \mathrm{~A}_{\mathrm{g}}^{-}, N\right)$ (gray symbols in Fig. 11) is larger than the percentages $T(s, N)$ 


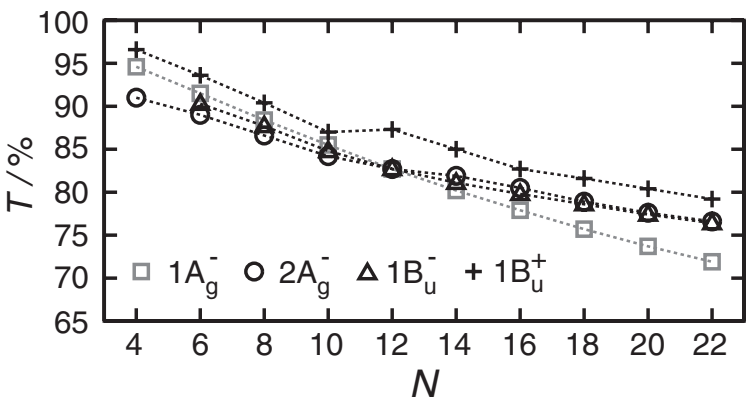

FIG. 11. Total contributions $T(s, N)$ of the $|R(s, N)|$ (cf. Table S15) most important SACs to the OM2/QCI $(\pi)$ wave functions of the polyenes with $N$ $\pi$-electrons and $s \in\left\{1 \mathrm{~A}_{\mathrm{g}}^{-}, 2 \mathrm{~A}_{\mathrm{g}}^{-}, 1 \mathrm{~B}_{\mathrm{u}}^{-}, 1 \mathrm{~B}_{\mathrm{u}}^{+}\right\}$.

of the covalent excitations as long as $N<12$. For $N>12$, however, the HF determinants cover percentages $T\left(1 \mathrm{~A}_{\mathrm{g}}^{-}, N\right)$ of the $\mathrm{OM} 2 / \mathrm{QCI}(\pi)$ ground state wave functions, which become increasingly smaller than the percentages $T(s, N)$ of the excited states (black symbols).

The latter finding indicates that the zeroth order description of the ground state by a single reference leads for $N>12$ to an increasingly inferior description of the ground state correlations compared to that of the excited states. To arrive at a more balanced description we tried a modified procedure for selecting the ground state reference SACs by requiring that the number $\left|R\left(1 \mathrm{~A}_{\mathrm{g}}^{-}, N\right)\right|$ should be chosen such that $T\left(1 \mathrm{~A}_{\mathrm{g}}^{-}, N\right)$ $\geq T\left(2 \mathrm{~A}_{\mathrm{g}}^{-}, N\right)$ at all $N$.

As shown by Table S16 in the SM, this prescription leads for $N \geq 12$ to increasing numbers $\left|R\left(1 \mathrm{~A}_{\mathrm{g}}^{-}, N\right)\right|$. A corresponding $\mathrm{MRCI}_{\mathrm{S}}$ treatment of the $1 \mathrm{~A}_{\mathrm{g}}^{-}$state yields then for $N \geq 12$ corrections $\Delta \tilde{E}_{0}(N)$ of the ground state energy. With these corrections we obtain the modified best estimates $\Delta \tilde{E}_{\mathrm{c},(\sigma, \pi)}(s, N)$ of the polyene spectra shown in Fig. S23 of the SM. This figure reveals a nearly perfect agreement of $\Delta \tilde{E}_{\mathrm{c},(\sigma, \pi)}\left(1 \mathrm{~B}_{\mathrm{u}}^{+}, N\right)$ with the best estimates of the experimental data that can be summarized by a RMSD of only $0.04 \mathrm{eV}$. In particular, the slight underestimate of the experimental $1 \mathrm{~B}_{\mathrm{u}}^{+}$ excitation energies visible for $N \geq 12$ in Fig. 10(a) is now essentially gone and the description of the $1 \mathrm{~A}_{\mathrm{g}}^{-}$and $1 \mathrm{~B}_{\mathrm{u}}^{+}$states has apparently become balanced.

Furthermore, for these two states requirement (ii) that $M(s, N)$ should vanish is fulfilled. Thus, the MRCI assumption of a cancellation of size-extensiveness errors is valid for

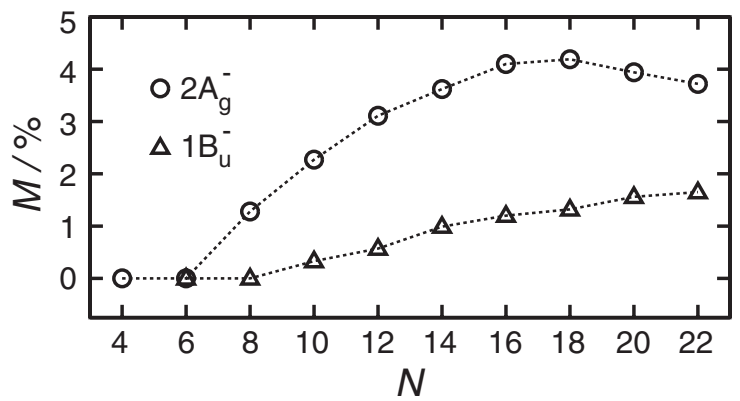

FIG. 12. Contributions $M(s, N)$ of the triply and quadruply excited SACs among the $|R(s, N)|$ (cf. Table S15) most important SACs to the OM2/QCI $(\pi)$ wave functions for two covalent excitations. these two states and the OM2/MRCI excitation energies of the $1 \mathrm{~B}_{\mathrm{u}}^{+}$state are very accurate (cf. Fig. S23).

\section{B. Causes and effects of lacking size consistency}

Figure 12 provides for the two covalent excitations $s \in\left\{2 \mathrm{~A}_{\mathrm{g}}^{-}, 1 \mathrm{~B}_{\mathrm{u}}^{-}\right\}$the check of the MRCI assumption (ii). The figure displays for these states the contributions $M(s, N)$ of triply and quadruply excited SACs among the $|R(s, N)|$ most important configurations to the respective QCI wave functions. These triply and quadruply excited SACs are missing in the reference sets $R(s, N)$ employed in our MRCI methods.

One recognizes in Fig. 12 that the contribution $M(s, N)$ of important triples and quadruples starts to become non-zero at $N \geq 8$ for the $2 \mathrm{~A}_{\mathrm{g}}^{-}$state and at $N \geq 10$ for the $1 \mathrm{~B}_{\mathrm{u}}^{-}$state, respectively. In the shorter polyenes the important higher than doubly excited SACs are exclusively triples. Important quadruples are first found at $N \geq 12\left(2 \mathrm{~A}_{\mathrm{g}}^{-}\right)$and $N \geq 14\left(1 \mathrm{~B}_{\mathrm{u}}^{-}\right)$, respectively. Here, a balanced and size-consistent MRCI description would require the inclusion of triply and quadruply excited SACs into the references sets $R\left(2 \mathrm{~A}_{\mathrm{g}}^{-}, N\right)$ and $R\left(1 \mathrm{~B}_{\mathrm{u}}^{-}, N\right)$, which then would lead to the appearance of quintuply and hextuply excited SACs in the MRCI bases of the covalent excitations. Because our current MRCI code cannot (yet) cope with higher than quadruply excited SACs, our MRCI computation of transition energies inherits, for the covalent excited states, a part of those errors that are caused by the lacking size-extensiveness ${ }^{55}$ of CI expansions truncated at a predefined excitation level.

The resulting size-inconsistency of our MRCI approach concerning the covalent excitations is highlighted by Fig. 13(a), which compares the experimental evidence (cf. Sec. I B in the SM) on the energy gap $\Delta E_{\mathrm{BA}}(N)=E\left(1 \mathrm{~B}_{\mathrm{u}}^{+}, N\right)$ - $E\left(2 \mathrm{~A}_{\mathrm{g}}^{-}, N\right)$ with our $\mathrm{MRCI}_{\mathrm{c}}$ predictions for the OM2
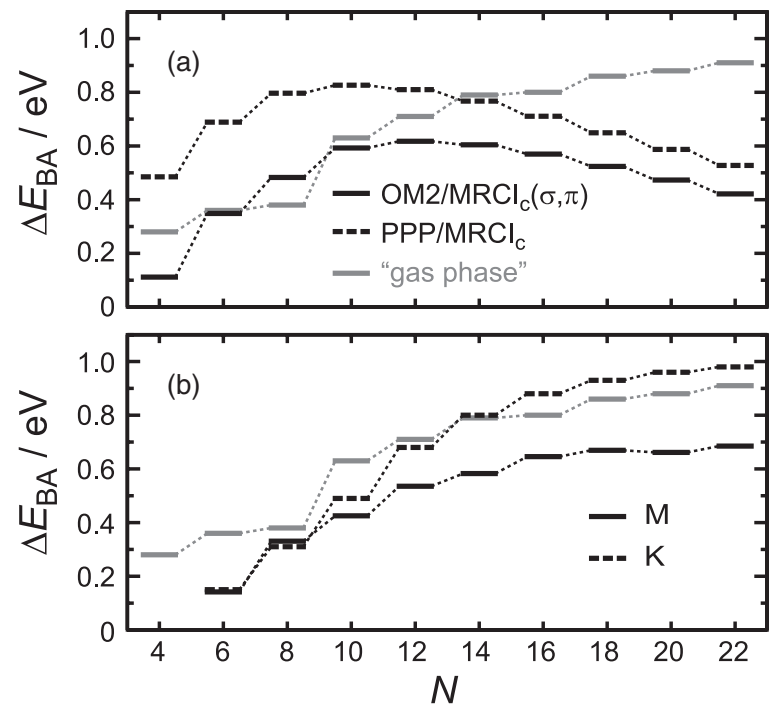

FIG. 13. Various theoretical predictions (black) of the energy gaps $\Delta E_{\mathrm{BA}}(N)$ between the ionic $1 \mathrm{~B}_{\mathrm{u}}^{+}$and the covalent $2 \mathrm{~A}_{\mathrm{g}}^{-}$excitations are compared with our best estimate of the vertical "gas phase" transition (gray, cf. Sec. I B in the SM). (a) $\mathrm{OM} 2 / \mathrm{MRCI}_{\mathrm{c}}(\sigma, \pi)$ (solid) and PPP/MRCI $\mathrm{C}_{\mathrm{c}}$ (dashed) from our work. (b) Theoretical predictions by Kurashige et al. ${ }^{43}$ (dashed) and by Marian et al. $^{45}$ (solid; numerical values extracted from Fig. 6, p. 1510). 
and PPP models of the polyenes. Experimentally, the gap $\Delta E_{\mathrm{BA}}(N)$ is seen to increase monotonously with growing polyene size $N$ and to approach a limiting value for very large polyenes. The $\mathrm{MRCI}_{\mathrm{c}}$ predictions, in contrast, erroneously assign decreasing gaps to the longer polyenes, while more (OM2) or less (PPP) correctly covering the increase of $\Delta E_{\mathrm{BA}}(N)$ up to $N=10(\mathrm{PPP})$ and $N=12(\mathrm{OM} 2)$. Because the electron correlations in the $1 \mathrm{~A}_{\mathrm{g}}^{-}$and $1 \mathrm{~B}_{\mathrm{u}}^{+}$state are covered in a balanced fashion implying excellent descriptions of the $1 \mathrm{~B}_{\mathrm{u}}^{+}$excitation energies (cf. Figs. 10 and S23), the decrease of $\Delta E_{\mathrm{BA}}(N)$ at large $N$ is exclusively due to the imbalanced and, therefore, size-inconsistent description of the $2 \mathrm{~A}_{\mathrm{g}}^{-}$state predicted by Fig. 12.

As a result the apparent success of OM2/MRCI concerning the $1 \mathrm{~B}_{\mathrm{u}}^{+}$excitation for $4 \leq N \leq 22$ suggests that the expansion of the reference sets required by Fig. 12 should be capable to remove the apparent size-consistency problem also for the $2 \mathrm{~A}_{\mathrm{g}}^{-}$and $1 \mathrm{~B}_{\mathrm{u}}^{-}$states and lead to correct gaps $\Delta E_{\mathrm{BA}}(N)$ over a much wider range of polyene sizes $N$.

The above analysis is supported by Fig. 13(b), which shows predictions on the gap $\Delta E_{\mathrm{BA}}(N)$ obtained by other authors, ${ }^{43,45}$ who employed different correlation treatments, which apparently were not (black dashed curve) or hardly (black solid curve) affected by the size-consistency problem, because both curves show (nearly) monotonous increases of the gap within the depicted range of polyene sizes.

In the case of the dashed curve ${ }^{43}$ the monotonicity is a necessary consequence of the applied correlation treatment, which consists for $N>10$ of a complete CI within a restricted $5 \times 5$ active space made up of the five energetically lowest occupied and the five energetically highest virtual $\pi$-MOs. For $N<10$ they chose a complete CI among all $\pi$-MOs $(\sigma-\pi$ interactions were approximately treated by perturbation theory). Such treatments are size-consistent, of course. On the other hand, the restriction of the active space to a constant size could lead to a neglect of important correlations in the longer compounds and the perturbational treatment to errors concerning the $\sigma-\pi$ correlations.

We have checked whether the $|R(s, N)|$ most important contributions to the $\mathrm{OM} 2 / \mathrm{QCI}(\pi)$ wave functions of the lowenergy polyene singlet states contain SACs, which are outside the $5 \times 5$ active CI space characterized above. We found that this is, indeed, the case for the polyenes with $N \geq 14\left(2 \mathrm{~A}_{\mathrm{g}}^{-}\right)$ and $N \geq 12\left(1 \mathrm{~B}_{\mathrm{u}}^{-}\right)$, respectively. As a result, one expects certain inaccuracies in the treatment of Kurashige et al., ${ }^{43}$ which may be the cause for the too steep increase of the covalent gap with $N$ in Fig. 13(b) and for the too strong decrease of all excitation energies with $N$ displayed by Fig. S24(a) in the SM.

The solid curve ${ }^{45}$ in Fig. 13(b) stems from a DFT/MRCI treatment, ${ }^{46}$ whose references are only singly and doubly excited (like in our MRCI). In this case a more detailed analysis is precluded by the partially empirical procedures, by which DFT/MRCI tries to avoid double counting of dynamical correlations that are already included by the DFT. ${ }^{46}$ The method underestimates the energy gap $\Delta E_{\mathrm{BA}}(N)$ at all polyene sizes by about $0.2 \mathrm{eV}$. Concerning excitation energies (see Fig. S24(b) in the SM) it increasingly underestimates those of the $1 \mathrm{~B}_{\mathrm{u}}^{+}$states toward larger $N($ at $N=22$ by about $0.65 \mathrm{eV})$.
For $N \geq 12$ the DFT/MRCI calculations also underestimate those of the $2 \mathrm{~A}_{\mathrm{g}}^{-}$state (at $N=22$ by about $0.43 \mathrm{eV}$ ). Thus, it seems that DFT/MRCI considerably overestimates the dynamical correlations in the excited states as compared to those in the ground state and favors ionic over covalent excitations.

According to the comparison between the available experimental evidence and the theoretical predictions of Kurashige et ll. $^{43}$ and Marian et al. $^{45}$ on the polyene spectra depicted in Fig. S24 in the SM, the too steep decrease of the predicted excitation energies with the polyene length $N$ indicates that these computational approaches, despite their size consistency (Fig. 13(b)), apparently do not provide balanced treatments of the electron correlations in the ground and excited states of the polyenes. OM2/MRCI (Fig. S23 in the SM), in contrast, lacks size consistency for the covalent excitations in the long polyenes $(N \geq 12)$ but otherwise provides balanced and accurate descriptions.

For comparison with further results on excitation energies in long polyenes, which, however, only cover either the covalent $^{18,26}$ or the ionic ${ }^{56}$ excitations and were obtained by DMRG/CASSCF,${ }^{26}$ valence bond DFT, ${ }^{18} \mathrm{PPP} / \mathrm{DMRG},{ }^{56}$ and $\mathrm{PPP} / \mathrm{CCSD},{ }^{56}$ respectively, Sec. IV $\mathrm{B}$ of the SM provides graphical representations of corresponding data in Figs. S25 and S26 together with a discussion. While the excitation energies obtained by these treatments may be inferior compared to a properly extended (and yet to be implemented) $\mathrm{OM} 2 / \mathrm{MRCI}$, they are certainly valuable for the computation of other properties, e.g., PPP/CCSD for non-linear polyene polarizabilities. ${ }^{56}$

\section{SUMMARY AND OUTLOOK}

As a result of our comparisons with the predictions of other theoretical approaches and with the available experimental evidence it seems that we were on the right track with our OM2/MRCI approach toward a quantitative description of the low-lying excitations also in polyenes with more than $12 \pi$-electrons. We fell short of this goal solely because the reference sets were restricted to singles and doubles and the maximum degree of excitation to quadruples. For the covalent excitations in polyenes with $N \geq 12$ this restriction leads to overestimation of the transition energies that are increasing with $N$. But apart from this issue, the OM2/MRCI description turned out to be quite accurate in all polyenes for the ionic $1 \mathrm{~B}_{\mathrm{u}}^{+}$excitation and in the shorter polyenes also for the covalent excitations. Hence, an extension of the method toward the inclusion of triple and quadruple excitations into the reference sets and of quintuples and hextuples into the MRCI expansions should largely remedy the remaining inaccuracies.

At the same time, the accurate descriptions obtained particularly for the shorter polyenes by OM2/MRCI and the reasonably similar descriptions rendered by PPP/MRCI, which were shown to be due to partial cancellations of errors associated with the previous use of model instead of optimized geometries and with the neglect of $\sigma-\pi$ and $\sigma-\sigma$ interactions, explain the well-known success of the early PPP/MRCI descriptions. $^{7}$ In a sense it was fortunate that computational limitations did not allow the treatment of longer polyenes at that time (1986), because then the errors of the MRCI 
approach that are connected with its restriction to singly and doubly excited references could not spoil results. Instead, the excitations in the longer polyenes were accessed by extrapolations using the excitation energies in the shorter compounds as references and these excitation energies were quantitatively reasonable as we have demonstrated above.

On the other hand, the extension of the description from PPP/MRCI to OM2/MRCI has added the new insight that the breaking of the PAS by OM2 is extremely weak and even weaker than in other treatments (cf. Secs. III B and III E). Therefore, the oscillator strengths of the transitions to the $1 \mathrm{~B}_{\mathrm{u}}^{-}$ states are very small in almost all polyenes except for the compound at which the level switch with the $1 \mathrm{~B}_{\mathrm{u}}^{+}$occurs. According to our best estimates (see Fig. 10(a)) this switch occurs near $N=14$, which is in close agreement with the spectroscopic data of Snyder et al. ${ }^{36}$ and Wang et al. ${ }^{37}$ discussed in the Introduction.

Our analysis of the various MRCI procedures has additionally fixed the details of the methods that should be used in future OM2/MRCI studies of polyene excited states. Such calculations can provide insights into the effects of excited state geometry relaxation and the sizes of the 0-0 transition energies. Within the accuracy limits of the present calculations, this extension toward 0-0 transitions may follow the computational procedures used above. However, if for a substantially enhanced accuracy in the description of the covalent excited states, an extension of the MRCI to triply and quadruply excited references is inevitable.

\section{ACKNOWLEDGMENTS}

This work was supported by the Deutsche Forschungsgemeinschaft (Grant No. TA 1167-1).

\footnotetext{
${ }^{1}$ R. Pariser and R. G. Parr, J. Chem. Phys. 21, 466 (1953).

${ }^{2}$ J. A. Pople, Trans. Faraday Soc. 49, 1375 (1953).

${ }^{3}$ R. G. Parr and R. Pariser, J. Chem. Phys. 23, 711 (1955).

${ }^{4}$ J. Hubbard, Proc. R. Soc. London, Ser. A 276, 238 (1963).

${ }^{5}$ E. Lieb and F. Wu, Phys. Rev. Lett. 20, 1445 (1968).

${ }^{6}$ F. Woynarovich, J. Phys. C 16, 5293 (1983).

${ }^{7}$ P. Tavan and K. Schulten, Phys. Rev. B 36, 4337 (1987).

${ }^{8}$ P. Strodel and P. Tavan, J. Chem. Phys. 117, 4667 (2002).

${ }^{9} \mathrm{~W}$. Weber and W. Thiel, Theor. Chem. Acc. 103, 495 (2000).

${ }^{10}$ M. R. Silva-Junior and W. Thiel, J. Chem. Theory Comput. 6, 1546 (2010).

${ }^{11}$ P. Tavan and K. Schulten, J. Chem. Phys. 85, 6602 (1986).

${ }^{12}$ See the supplementary material at http://dx.doi.org/10.1063/1.3696880 which provides on 31 pages in four sections a total of thirteen figures (S14S26) and thirteen tables (S4-S16) additional material to (I) the introduction, (II) the applied method, (III) the computational results, and (IV) the summary and discussion.

${ }^{13}$ P. Tavan and K. Schulten, Mater. Res. Soc. Symp. Proc. 109, 163 (1988).

${ }^{14}$ J. Čížek, J. Paldus, and I. Hubač, Int. J. Quantum Chem. 8, 951 (1974).

${ }^{15}$ K. Schulten, I. Ohmine, and M. Karplus, J. Chem. Phys. 64, 4422 (1976).

${ }^{16}$ B. S. Hudson, B. E. Kohler, and K. Schulten, in Excited States, Vol. 6, edited by E. C. Lim (Academic Press, New York, 1982).
}

${ }^{17}$ Z. Soos and S. Ramasesha, Phys. Rev. B 29, 5410 (1984).

${ }^{18}$ W. Wu, D. Danovich, A. Shurki, and S. Shaik, J. Phys. Chem. A 104, 8744 (2000)

${ }^{19}$ R. Pariser, J. Chem. Phys. 24, 250 (1956).

${ }^{20}$ E. Hückel, Z. Phys. 70, 204 (1931).

${ }^{21}$ T. Polívka and V. Sundström, Chem. Rev. 104, 2021 (2004).

${ }^{22}$ H. Hashimoto, K. Yanagi, M. Yoshizawa, D. Polli, G. Cerullo, G. Lanzani, S. D. Silvestri, A. T. Gardiner, and R. J. Cogdell, Arch. Biochem. Biophys. 430, 61 (2004).

${ }^{23}$ Y. Koyama, Y. Kakitani, T. Miki, R. Christiana, and H. Nagae, Int. J. Mol. Sci. 11, 1888 (2010).

${ }^{24}$ G. Cerullo, D. Polli, G. Lanzani, S. D. Silvestri, H. Hashimoto, and R. J. Cogdell, Science 298, 2395 (2002).

${ }^{25}$ K. Nakayama, H. Nakano, and K. Hirao, Int. J. Quantum Chem. 66, 157 (1998).

${ }^{26}$ D. Ghosh, J. Hachmann, T. Yanai, and G. K. Chan, J. Chem. Phys. 128, 144117 (2008).

${ }^{27}$ D. Zhang and C. Liu, J. Chem. Phys. 135, 134117 (2011).

${ }^{28}$ K. Schulten and M. Karplus, Chem. Phys. Lett. 14, 305 (1972).

${ }^{29}$ B. S. Hudson and B. E. Kohler, Chem. Phys. Lett. 14, 299 (1972).

${ }^{30}$ R. J. Cave and E. R. Davidson, J. Phys. Chem. 92, 2173 (1988).

${ }^{31}$ L. Serrano-Andres, M. Merchan, I. Nebot-Gil, R. Lindh, and B. O. Roos, J. Chem. Phys. 98, 3151 (1993).

${ }^{32}$ L. Serrano-Andres, R. Lindh, B. O. Roos, and M. Merchan, J. Phys. Chem. 97, 9360 (1993).

${ }^{33}$ C. Angeli and M. Pastore, J. Chem. Phys. 134, 184302 (2011).

${ }^{34}$ T. Sashima, H. Nagae, M. Kuki, and Y. Koyama, Chem. Phys. Lett. 299, 187 (1999).

${ }^{35}$ Y. Koyama, F. S. Rondonuwu, R. Fujii, and Y. Watanabe, Biopolymers 74, 2 (2004).

${ }^{36}$ R. Snyder, E. Arvidson, C. Foote, L. Harrigan, and R. L. Christensen, J. Am. Chem. Soc. 107, 4117 (1985)

${ }^{37}$ P. Wang, R. Nakamura, Y. Kanematsu, Y. Koyama, H. Nagae, T. Nishio, H. Hashimoto, and J. Zhang, Chem. Phys. Lett. 410, 108 (2005).

${ }^{38}$ R. R. Chadwick, M. Z. Zgierski, and B. S. Hudson, J. Chem. Phys. 95, 7204 (1991).

${ }^{39}$ W. J. Buma, B. E. Kohler, and K. Song, J. Chem. Phys. 94, 6367 (1991).

${ }^{40}$ R. M. Gavin, S. Riesemberg, and S. A. Rice, J. Chem. Phys. 58, 3160 (1973).

${ }^{41}$ R. M. Gavin, C. Weisman, J. K. McVey, and S. A. Rice, J. Chem. Phys. 68, 522 (1978).

${ }^{42}$ R. L. Christensen, M. G.I. Galinato, E. F. Chu, J. N. Howard, R. D. Broene, and H. A. Frank, J. Phys. Chem. A 112, 12629 (2008).

${ }^{43}$ Y. Kurashige, H. Nakano, Y. Nakao, and K. Hirao, Chem. Phys. Lett. 400, 425 (2004).

${ }^{44}$ J. Starcke, M. Wormit, J. Schirmer, and A. Dreuw, Chem. Phys. 329, 39 (2006).

${ }^{45}$ C. M. Marian and N. Gilka, J. Chem. Theory Comput. 4, 1501 (2008).

${ }^{46}$ S. Grimme and M. Waletzke, J. Chem. Phys. 111, 5645 (1999).

${ }^{47}$ M. Schreiber, M. R. Silva-Junior, S. P.A. Sauer, and W. Thiel, J. Chem. Phys. 128, 134110 (2008).

${ }^{48}$ P. Strodel and P. Tavan, J. Chem. Phys. 117, 4677 (2002).

${ }^{49}$ P. Tavan and K. Schulten, J. Chem. Phys. 72, 3547 (1980).

${ }^{50} \mathrm{~W}$. Thiel, MNDO97, version 5.0, MPI für Kohlenforschung, Mülheim an der Ruhr, Germany, 1997.

${ }^{51}$ R. J. Cave and E. R. Davidson, J. Phys. Chem. 91, 4481 (1987).

${ }^{52}$ R. J. Cave and E. R. Davidson, J. Phys. Chem. 92, 614 (1988).

${ }^{53}$ C. Yannoni and T. Clarke, Phys. Rev. Lett. 51, 1191 (1983).

${ }^{54}$ W. Haugen and M. Trætteberg, Acta Chem. Scand. 20, 1726 (1966).

${ }^{55}$ A. Szabo and N. S. Ostlund, Modern Quantum Chemistry: Introduction to Advanced Electronic Structure Theory (Dover, New York, 1996).

${ }^{56}$ Z. Shuai and J. Brédas, Phys. Rev. B 62, 15452 (2000). 